九州大学学術情報リポジトリ

Kyushu University Institutional Repository

Rattleback dynamics and its reversal time of rotation

近藤，洋一郎

ht tps://doi. org/10.15017/1866252

出版情報：九州大学，2017，博士（理学），課程博士 バージョン：

権利関係 : 


\title{
Rattleback dynamics and its reversal time of rotation
}

\author{
Yoichiro Kondo \\ Department of Physics, Graduate School of Science, \\ Kyushu University, Japan
}

\author{
A thesis submitted for \\ the degree of Doctor of Science
}

2017 


\section{Abstract}

A rattleback is a rigid, semi-elliptic toy which exhibits unintuitive spinning behavior; when it is spun in one direction, it soon begins pitching and stops spinning, then it starts to spin in the opposite direction, but in the other direction, it seems to spin just steadily. This puzzling behavior results from the slight misalignment between the principal axes for the inertia and those for the curvature; the misalignment couples the spinning motion with the pitching and rolling oscillations.

In this thesis, we reformulate the rattleback dynamics under the no-slip condition and without dissipation by Garcia and Hubbard [Proc. R. Soc. Lond. A 418, 165 (1988)]. In the small spin and small oscillation regime, we reduce the dynamics to that of three variables, i.e. the spin, the pitching energy and the rolling energy, and they are coupled by the two coefficients called asymmetric torque coefficients. It has been shown that the spin can reverse in both directions, and using the simplified dynamics, we derive the formula for the time required for the spin reversal $t_{r}$, which has been originally obtained by Garcia and Hubbard. We show that (i) Garcia-Hubbard formula can be expressed in a simple form consisting of four factors, i.e. the misalignment angle, the difference in the inverses of inertia moment for the two oscillations, that in the radii for the two principal curvatures, and the squared frequency of the oscillation, and that (ii) the averaged torque generated by the pitching and that by the rolling always have opposite signs to each other. We then show that the ratio of the asymmetric torque coefficients, which determines the ratio of the times for reversal in the spinning direction, is simply given by the ratio of the squared oscillation frequencies. For a typical rattleback, the pitch frequency is significantly faster than the roll frequency, therefore the time for reversal in one direction is much shorter than that in the other spinning direction.

We also perform extensive numerical simulations to examine validity and limitation of Garcia-Hubbard formula for the time for spin reversal $t_{r}$. We find that (i) Garcia-Hubbard formula for $t_{r}$ is good for both the spinning directions in the small spin and small oscillation regime, and that (ii) in the 
fast spin regime especially for the steady direction, the rattleback may not reverse and shows a rich variety of dynamics including steady spinning, spin wobbling, and chaotic behavior reminiscent of chaos in a dissipative system.

Despite the fact that the simplified dynamics leads to the periodic behavior, after the first spin reversal time, our simulation results demonstrate that the system shows quite rich dynamics: quasi-periodic behavior, chaotic behavior, and quasi-periodic yet the periods being significantly shorter than the first one. We discuss this breakdown of periodicity in connection with breakdown of approximations/assumptions used in deriving the simplified dynamics. 


\section{Contents}

1 Introduction $\quad 1$

1.1 What is rattleback? . . . . . . . . . . . . . . . 1

1.2 An overview of the literature . . . . . . . . . . . . . 3

1.2.1 On rattleback dynamics . . . . . . . . . . . . 3

1.2.2 Related topics in physics . . . . . . . . . . . . 4

1.3 Motivation and outline of this thesis . . . . . . . . 5

2 Theory $\quad 7$

2.1 Model equations . . . . . . . . . . . . . . . . . . . 7

2.1.1 Equations of motion . . . . . . . . . . . . . . 7

2.1.2 Rattleback parameters . . . . . . . . . . . . . . 10

2.1.3 On averaged torque and chirality . . . . . . . . . 10

2.2 Small amplitude approximation of oscillations under $\omega_{z}=0$. . 11

2.3 Garcia and Hubbard's theory for the time for reversal . . . . . 13

2.3.1 Asymmetric torque coefficients . . . . . . . . . . . 13

2.3.2 Typical rattleback parameters . . . . . . . . . . . . 15

2.3.3 Time for reversal . . . . . . . . . . . . . . . 16

2.3.4 Parameter dependences of the time for reversal . . . . 18

2.4 Three zones of the parameter space . . . . . . . . . . . . . 19

2.5 Oscillations with a small constant spin . . . . . . . . . 20

2.6 Comparison of our theory with related works . . . . . . . . . . 23

3 Simulation $\quad 25$

3.1 Shell-dumbbell model . . . . . . . . . . . . . . . . . . 25

3.2 Simulation methods . . . . . . . . . . . . . . . . . 26

3.3 Simulation results for the parameter set GH . . . . . . . . 28

3.3.1 General behavior for the parameter set GH . . . . . . 28

3.3.2 Comparison with Garcia-Hubbard formulae . . . . . . 30

3.4 Simulation with the parameter set SD . . . . . . . . . . 31

3.4.1 Unsteady initial spin direction $\left(n_{i}>0\right) \ldots \ldots . \ldots 34$

3.4.2 Steady initial spin direction $\left(n_{i}<0\right) \ldots \ldots . \ldots 34$ 
4 Summary and Discussion 39

4.1 Summary of our work . . . . . . . . . . . . . . . . . 39

4.2 Discussions . . . . . . . . . . . . . . . . . 40

4.2.1 After the first round of spin reversals . . . . . . . . . 40

4.2.2 Pippard's rattleback revisited . . . . . . . . . . . . 42

4.2.3 On elliptic and semi-elliptic rattlebacks . . . . . . . . . 42

4.2.4 Atypical choices of parameters . . . . . . . . . . . . . . 43

4.2 .5 On initial conditions . . . . . . . . . . . . . . . 44

4.3 Concluding remarks . . . . . . . . . . . . . . . . 45

A Rattleback parameters for semi-ellipsoids 47

B Equivalence of $K_{p}$ and $K_{r}$ with the original expressions $\quad 49$

$\begin{array}{ll}\text { Bibliography } & 51\end{array}$ 


\section{List of variables}

\begin{tabular}{c|c|c}
\hline $\boldsymbol{u}$ & vertical unit vector pointing upward & Eq. $(2.1)$ \\
$\boldsymbol{\omega}$ & angular velocity around the center of mass G & Eq. $(2.2)$ \\
$\boldsymbol{r}$ & vector from G to the contact point & Eq. $(2.2)$ \\
$a$ & static height of the center of mass & Eq. $(2.5)$ \\
$\theta, \phi$ & dimensionless principal curvatures & Eq. $(2.5)$ \\
$\xi$ & skew angle & Eq. $(2.5)$ \\
$\alpha, \beta, \gamma$ & dimensionless principal moments of inertia & Eq. $(2.15)$ \\
$\omega_{p}, \omega_{r}$ & pitch frequency and roll frequency & Eq. $(2.26)$ \\
$K_{p}, K_{r}$ & asymmetric torque coefficients & Eqs. $(2.44),(2.45)$ \\
$n$ & spin (vertical angular velocity) & Eq. $(2.51)$ \\
$n_{i}$ & initial spin & Eq. $(2.57)$ \\
$t_{r G H \pm}$ & Garcia-Hubbard formulae for time for reversal & Eqs. $(2.61),(2.63)$ \\
$t_{r u}, t_{r s}$ & simulated time for reversal & - \\
$\left|\omega_{x y 0}\right|$ & initial oscillation amplitude & Eq. (3.9) \\
\hline
\end{tabular}




\section{Chapter 1}

\section{Introduction}

\subsection{What is rattleback?}

A rattleback, also called as a celt, celtic stone, or wobble stone, is a spinning toy which exhibits unintuitive dynamical behavior. Commercially available rattlebacks are mostly (but not always) boat-shaped, semi-elliptic rigid objects made of plastic or wood (Fig.1.1). It spins smoothly when spun in one direction, however, when spun in the other direction, it soon starts wobbling or rattling about its short axis and stops spinning, then it starts to rotate in the opposite direction. One who has studied classical mechanics must be amazed by this reversal in spinning, because it apparently seems to violate the angular momentum conservation and the chirality emerges from a seemingly symmetrical object.

Although rattlebacks are often classified as spinning tops [1, 2, 3], there is a big difference between them, i.e., rattlebacks do not need to be spun fast. For a familiar top to keep spinning, fast spin is necessary to generate the gyroscopic effect, which keeps it from falling. Also, the torque due to slip at the contact point lifts up the center of mass [4]. By contrast, a rattleback is statically stable, and only one or two revolutions per second is enough to observe the spin reversal. Therefore, we can guess that the gyroscopic effect and the torque due to slip are not crucial for the spin reversal behavior.

Historically, stones with this reversal effect, especially ones found in ancient remains, are called as celts (Fig.1.1). The name has nothing to do with the Celts, an ancient tribe in Europe. The word "celte" in the Latin Bible was interpreted as a chisel, and then the word has become the name of chiral stones [5]. After a physicist Jeal Walker introduced celts under the name of rattleback in Scientific American in 1979, "rattleback" has become a popular name [2]. We use the name rattleback throughout this thesis. 

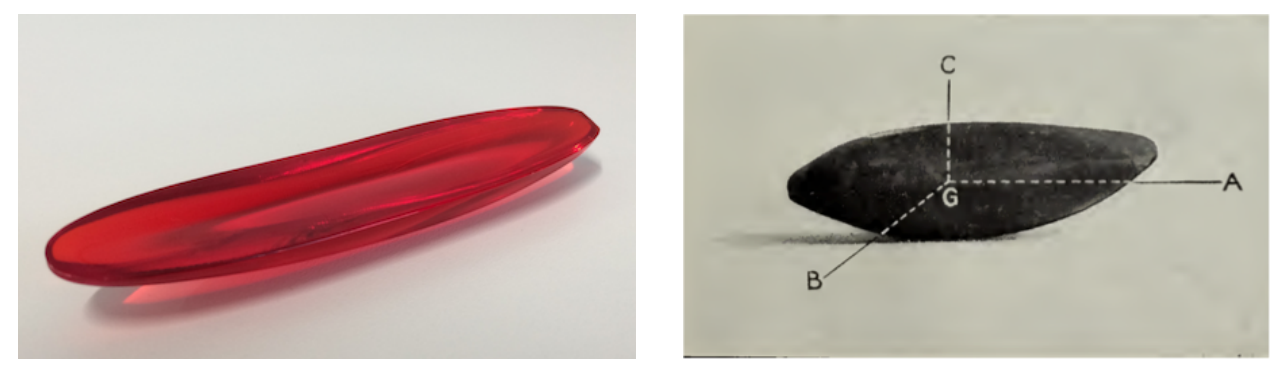

Figure 1.1: (left) A commercially available rattleback made of plastic. (right) A celt stone, from the book on spinning tops, written in 1909 [3].

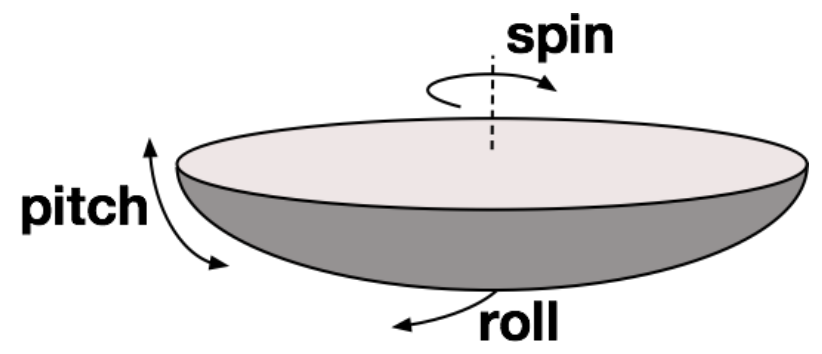

Figure 1.2: A schematic illustration of pitching, rolling and spinning.

There are three requirements for a rattleback to show this reversal of rotation; (i) the two principal curvatures of the lower surface should be different, (ii) the two horizontal principal moments of inertia should also be different, and (iii) the principal axes of inertia should be misaligned to the principal directions of curvature. These characteristics induce the coupling between the spinning motion and the two oscillations: the pitching about the short horizontal axis and the rolling about the long horizontal axis (Fig. 1.2). The coupling is asymmetric, i.e., the oscillations cause torque around the spin axis and the signs of the torque are opposite to each other. This also means that either the pitching or the rolling is excited depending on the direction of the spinning. We will see that the spinning motion couples with the pitching much stronger than that with the rolling, therefore, it takes much longer time for spin reversal in one direction than in the other direction; that is why most rattlebacks reverse only for one way before they stop by dissipation.

Objects which satisfy these requirements are not rare at all. In fact, we can make rattlebacks by bending a spoon, putting some weights on a classic phone receiver, and so on (Fig. 1.3). Various photos and movies of rattlebacks can be found on the Internet. Among them, we mention a rattleback made of a wine bottle, which was made by Pippard, a prominent physicist [6]. He cut gently tapered part of a wine bottle and glued a heavy 

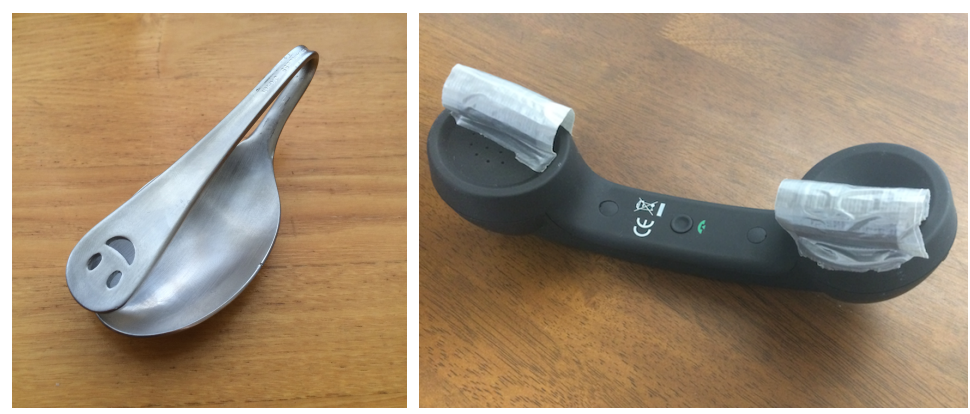

Figure 1.3: A bended spoon (left) and a phone receiver with two weights (right) that show reversals of rotation.

brass bar on it. This rattleback reverses its spin several times when it is spun at about one revolution per second. He noted that to make a "good" rattleback, two radii of curvature should differ significantly $(3.5 \mathrm{~cm}$ and $25 \mathrm{~cm}$ in his case), the friction between a horizontal surface and a rattleback should be large so that it rolls on the surface rather than slips, a long inertia bar is desirable, and a contact area (ideally a point) should be small to prevent dumping. These know-hows are examined later in the thesis.

\subsection{An overview of the literature}

\subsubsection{On rattleback dynamics}

In the 1890s, a meteorologist Walker performed the first quantitative analysis of the rattleback motion [7, 1]. He is most known today for Walker Circulation, i.e., an atmospheric circulation on the Pacific Ocean. His works were performed at Cambridge before he moved to India, where he published pioneering works on the modern meteorology [8]. In [1], he linearized the equations of motion under the assumptions that the rattleback does not slip at the contact point and that the rate of spinning speed changes much slower than other time scales, and showed that either the pitching or the rolling becomes unstable depending on the direction of the spin.

More rigorous conditions under which the instabilities develop were determined by Bondi [9], who is known for the steady state universe, and recently by Wakasugi [10]. Case and Jalal [11] derived the growth rate of instability at small rotational speed based on Bondi's formulation. Markeev [12], Pascal [13], and Blackowiak et al. [14] obtained equations including the spin mode, namely the equations which show the reversal of rotation, by extracting the slowly varying amplitudes of the fast oscillations (pitching and rolling). Mof- 
fatt and Tokieda [15] derived similar equations to those of [12] and [13], and pointed out the analogy to the $\alpha \omega$-dynamo theory, which explains the dynamo action in the inner earth; in fact, the geomagnetic field is also known to change its direction. Garcia and Hubbard [16] obtained the analytical formulae of the averaged torques induced by pure pitching and rolling, which are then used to describe the spin evolution. In the next chapter, we review Bondi [9], Case and Jalal [11], and Moffatt and Tokieda [15], and compare these works with our theory, which is based on Garcia and Hubbard [16].

In the 1980s, as the first numerical study, Kane and Levinson [17] simulated the energy-conserving equations and showed that the rattleback changes its spinning direction indefinitely for certain parameter values and initial conditions. They also showed that it starts to rotate when begun with only pure pitching or rolling, but the direction of the rotation is different between pitching and rolling. Similar simulations were performed by Lindberg Jr. and Longman independently [18]. Nanda et al. recently simulated the spin resonance of the rattleback on a vibrating base [19].

Energy conserving dynamical systems usually conserve the phase volume, but the present rattleback dynamics does not explore the whole phase volume with a given energy because of the no-slip condition, which is a non-holonomic constraint. Therefore, Liouville theorem does not hold, and such a system has been shown to behave much like dissipative systems. Borisov and Mamaev in fact reported the existence of "strange attractor" for certain parameter values in the present system [20]. The no-slip rattleback system has been actively studied in the context of chaotic dynamics during the last decade [21, 22, $23,24,25]$. We will also encounter chaotic behaviors in our simulations in Chapter 3.

Effects of dissipation at the contact point have been investigated in several works. Magnus [26] and Karapetyan [27] incorporated a viscous type of friction to the equations. Takano [28] determined the conditions under which the reversal of rotation occurs with the viscous dissipation. Garcia and Hubbard [16] simulated equations with aerodynamic force, Coulomb friction due to spinning, and dissipation due to slippage; then they compared the results with a real rattleback. The dissipative rattleback models based on the contact mechanics with Coulomb friction have been developed by Zhuravlev et al. [29], Kudra and Awrejcewicz [30, 31, 32].

\subsubsection{Related topics in physics}

Spinning motions of rigid bodies have been studied for centuries, from the rotaion of the earth to the exactly solvable spinning tops, and still are drawing interest in recent years. Moffatt investigated the dynamics of a heavy thick 
disk called Euler's disk [33]. During the spinning process, the disk makes a noise with its frequency increasing, then it abruptly stops. He made a simple model of Euler's disk by simply incorporating no-slip dynamics with aerodynamic dissipation, and showed the divergence of the frequency and the breakdown of the theory just before it stops. Recently, an interesting dynamics of rolling rings [34] was investigated. Unlike Euler's disk, air can move freely through the hole of the ring, and under the aerodynamic dissipation, this makes the sign of ring's orbital angular momentum changes just before it stops. Moffatt and Shimomura revisited a well known phenomena of spinning eggs $[35,36]$. They explained why an egg rises when it is spun fast, and they found that an egg can even jump [37, 38]. In this case, dissipation due to slip plays a crucial role as classical tippe tops. For all these works, the spinning speed needs to be fast enough and dissipation mechanism is essentially required, in contrast to the rattleback dynamics we will investigate in the present thesis.

Rattleback dynamics can also be viewed as a mechanical system which converts oscillation to rotation and vise versa. Not to mention that such a system is important for engineering purposes, this coupling has also been studied in various contexts in physics such as a circular granular ratchet [39], and bouncing dumbbells, which shows a cascade of bifurcations [40].

\subsection{Motivation and outline of this thesis}

Although the previous works we have briefly reviewed can answer the general mechanism of the spin reversal behavior, differences of motions among various rattlebacks have not been sufficiently investigated. In fact, some rattlebacks such as Pippard's rattleback we have mentioned show relatively strong coupling between spinning and rolling than that of Fig. 1.1; what factors create the difference among them has not been fully explained.

In addition to that, recent developments in 3D printing technology have paved a way to make complicated 3D-objects with controlled mass distributions. Bächer et al. [41] proposed a novel algorithm to make a complicated $3 \mathrm{D}$ object which spins stably by optimizing the mass distribution inside it. Understanding the effects of inertial and shape factors on the rattleback motion may enable us to create new rattleback designs by using such technology.

Based on these motivations, in the present thesis, we study the minimal model for the rattleback dynamics, i.e., a spinning rigid body with a no-slip contact ignoring any form of dissipation.

This thesis is organized as follows. Theoretical part is given in the next chapter. We reformulate Garcia and Hubbard's theory [16] of rattleback 
dynamics under the no-slip and no-dissipation conditions, and reduced it to that of three variables, i.e. the spin, the pitching energy and the rolling energy, in the small spin and small oscillation regime. We then focus on the time required for reversal, or what we call the time for reversal, which is the most evident quantity that characterizes rattlebacks, and obtain a concise expression for Garcia-Hubbard formula for the time for reversal [16]. Related works are reviewed and compared with our theory. In Chapter 3 we give simulation results for the time for reversal. After we show typical simulated spin behaviors, we investigate how the time for reversal depends on the various model parameters and initial conditions by numerical simulations to examine the validity and the limitation of the theory. Summary and discussion are given in Chapter 4. 


\section{Chapter 2}

\section{Theory}

In the first section of this chapter, we formulate the equations of motion of the rattleback and the constraints we investigate throughout this thesis. After linearizing the equations in the small oscillations under the zero-spin regime in Section 2.2, we reformulate Garcia and Hubbard's theory for the time for reversal [16] in Section 2.3. We then review related works by Bondi [9] and Case and Jalal [11] in Section 2.4 and 2.5, respectively. Comparison of our theory with related works is presented in the last section.

\subsection{Model equations}

\subsubsection{Equations of motion}

We consider a rattleback as a rigid body, whose configuration can be represented by the position of the center of mass $G$ and the Euler angles; both of them are obtained by integrating the velocity of the center of mass $\boldsymbol{v}$ and the angular velocity $\boldsymbol{\omega}$ around it [42].

We investigate the rattleback motion on a horizontal plane, assuming that it is always in contact with the plane at a single point $\mathrm{C}$ without slipping. We ignore dissipation, then all the forces that act on the rattleback are the contact force $\boldsymbol{F}$ exerted by the plane at $\mathrm{C}$ and the gravitational force $-M g \boldsymbol{u}$, where $\boldsymbol{u}$ represents the unit vertical vector pointing upward (Fig. 2.1). Therefore, the equations of motion are given by

$$
\begin{aligned}
& \frac{d(M \boldsymbol{v})}{d t}=\boldsymbol{F}-M g \boldsymbol{u}, \\
& \frac{d(\hat{I} \boldsymbol{\omega})}{d t}=\boldsymbol{r} \times \boldsymbol{F},
\end{aligned}
$$




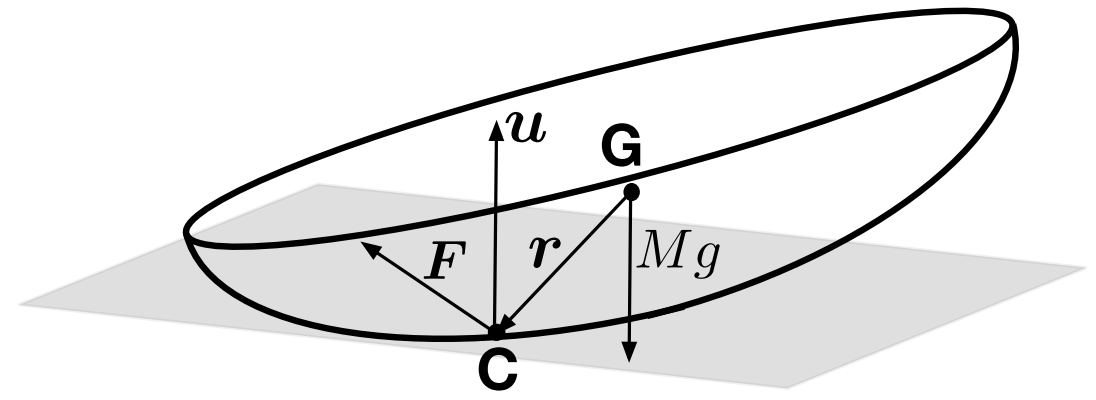

Figure 2.1: Notations of the rattleback.

where $M$ and $\hat{I}$ are the mass and the inertia tensor around G, respectively, and $\boldsymbol{r}$ is the vector from $\mathrm{G}$ to the contact point $\mathrm{C}$.

The contact force $\boldsymbol{F}$ is determined by the conditions of the contact point; our assumptions are that (i) the rattleback is always in contact at a point with the plane, and (ii) there is no-slip at the contact point. The second constraint is represented by the relation by which $\boldsymbol{v}$ is related with $\boldsymbol{\omega}$ as

$$
\boldsymbol{v}=\boldsymbol{r} \times \boldsymbol{\omega}
$$

Before formulating the constraint (i), we specify the co-ordinate system. We employ the body-fixed co-ordinate with the origin being the center of mass $\mathrm{G}$, and the axes being the principal axes of inertia; the $z$-axis is the one close to the spinning axis pointing downward, and the $x$ and $y$ axes are taken to be $I_{x x}>I_{y y}$ (Fig. 2.2).

In this co-ordinate, the lower surface function of the rattleback is assumed to be given by

$$
f(x, y, z)=0
$$

where

$$
f(x, y, z) \equiv \frac{z}{a}-1+\frac{1}{2 a^{2}}(x, y) \hat{R}(\xi) \hat{\Theta} \hat{R}^{-1}(\xi)\left(\begin{array}{l}
x \\
y
\end{array}\right)
$$

with

$$
\hat{R}(\xi) \equiv\left(\begin{array}{cc}
\cos \xi, & -\sin \xi \\
\sin \xi, & \cos \xi
\end{array}\right), \quad \hat{\Theta} \equiv\left(\begin{array}{cc}
\theta, & 0 \\
0 & \phi
\end{array}\right)
$$

Here $a$ is the distance between $\mathrm{G}$ and the surface at $x=y=0$, and $\xi$ is the skew angle by which the principal directions of curvature are rotated from the $x-y$ axes, which we choose as the principal axes of inertia (Fig. 2.2). $\theta / a$ and $\phi / a$ are the principal curvatures at the bottom, namely at $(0,0, a)^{t}$. 
Now, we can formulate the contact point condition (i); the components of the contact point vector $\boldsymbol{r}$ should satisfy Eq. (2.4), and the normal vector of the surface at $\mathrm{C}$ should be parallel to the vertical vector $\boldsymbol{u}$. Thus we have

$$
\boldsymbol{u} \| \nabla f
$$

which gives the relation

$$
\frac{\boldsymbol{r}_{\perp}}{a}=\frac{1}{u_{z}} \hat{R}(\xi) \hat{\Theta}^{-1} \hat{R}^{-1}(\xi) \boldsymbol{u}_{\perp},
$$

where $\boldsymbol{a}_{\perp}$ represents the $x$ and $y$ components of a vector $\boldsymbol{a}$ in the body-fixed co-ordinate.

Before we proceed, we introduce a dotted derivative $\dot{\boldsymbol{a}}$ of a vector $\boldsymbol{a}$ defined as the time derivative of the vector components in the body-fixed co-ordinate. This is related to the time derivative by

$$
\frac{d \boldsymbol{a}}{d t}=\dot{\boldsymbol{a}}+\boldsymbol{\omega} \times \boldsymbol{a} .
$$

Note that the vertical vector $\boldsymbol{u}$ does not depend on time, thus we have

$$
\frac{d \boldsymbol{u}}{d t}=\dot{\boldsymbol{u}}+\boldsymbol{\omega} \times \boldsymbol{u}=\mathbf{0} .
$$

These conditions, i.e., the no-slip condition Eq. (2.3), the conditions of the contact point Eqs. (2.4) and (2.8), and the vertical vector condition Eq. (2.10) close the equations of motion Eqs. (2.1) and (2.2).

We remark two properties of the present system. First, there exists the energy integral:

$$
E=\frac{1}{2} \boldsymbol{\omega} \cdot(I \boldsymbol{\omega})+\frac{1}{2} M v^{2}+M g \boldsymbol{u} \cdot \boldsymbol{r}=\text { const. }
$$

Second, the equations are invariant under time reversal, i.e. the equations are unchanged under the transformation $t \rightarrow-t$ and $\boldsymbol{\omega} \rightarrow-\boldsymbol{\omega}$.

Following Garcia and Hubbard [16], we describe the rattleback dynamics by $\boldsymbol{u}$ and $\boldsymbol{\omega}$. The evolution of $\boldsymbol{\omega}$ is obtained as

$$
\begin{aligned}
\hat{I} \dot{\boldsymbol{\omega}}-M \boldsymbol{r} \times(\boldsymbol{r} \times \dot{\boldsymbol{\omega}})= & -\boldsymbol{\omega} \times(\hat{I} \boldsymbol{\omega}) \\
& +M \boldsymbol{r} \times(\dot{\boldsymbol{r}} \times \boldsymbol{\omega}+\boldsymbol{\omega} \times(\boldsymbol{r} \times \boldsymbol{\omega}))+M g \boldsymbol{r} \times \boldsymbol{u}
\end{aligned}
$$

by eliminating the contact force $\boldsymbol{F}$ from the equations of motion (2.1) and (2.2), and using the no-slip condition (2.3). The state variables $\boldsymbol{u}$ and $\boldsymbol{\omega}$ can be determined by Eqs. (2.10) and (2.12) with the contact point conditions Eqs. (2.4) and (2.8). Since $\boldsymbol{u}$ is a unit vector, the present system has five degrees of freedom. 


\subsubsection{Rattleback parameters}

The rattleback is characterized by the inertial parameters $M, I_{x x}, I_{y y}, I_{z z}$, the geometrical parameters $\theta, \phi, a$, and the skew angle $\xi$. For the stability of the rattleback, both of the dimensionless curvatures $\theta$ and $\phi$ should be smaller than 1; without loss of generality, we assume

$$
0<\phi<\theta<1
$$

then, it is enough to consider

$$
-\frac{\pi}{2}<\xi<0
$$

for the range of the skew angle $\xi$. The positive $\xi$ case can be obtained by the reflection with respect to the $x$ - $z$ plane.

At this stage, we introduce the dimensionless inertial parameters $\alpha, \beta$, and $\gamma$ for later use after Bondi [9] as

$$
\alpha \equiv \frac{I_{x x}}{M a^{2}}+1, \beta \equiv \frac{I_{y y}}{M a^{2}}+1, \gamma \equiv \frac{I_{z z}}{M a^{2}},
$$

which are dimensionless inertial moments at the contact point C. Note that

$$
\alpha>\beta>1
$$

because we have assumed $I_{x x}>I_{y y}$.

\subsubsection{On averaged torque and chirality}

A big surprise of the rattleback motion is its apparent violation of the conservation law of the vertical angular momentum. The torque around the vertical axis, which is responsible for the reversal, is given by

$$
\boldsymbol{u} \cdot(\boldsymbol{r} \times \boldsymbol{F})=M(\boldsymbol{u} \times \boldsymbol{r}) \cdot \frac{d \boldsymbol{v}}{d t} .
$$

Note that the contact point vector $\boldsymbol{r}$ is generally not parallel to $\boldsymbol{u}$ because the bottom surface is not spherical, thus the torque around the vertical axis does not need to be zero on average. We estimate this averaged torque later in this chapter.

Another surprise is its chiral behavior, namely the apparently symmetrical rattleback shows different behaviors depending on the spinning directions. This chiral behavior results from the skew angle $\xi$, and $|\xi| \ll 1$ for typical rattlebacks; the chiral symmetry breaking is ingeniously hidden in the rattleback, which makes the motion counterintuitive. 


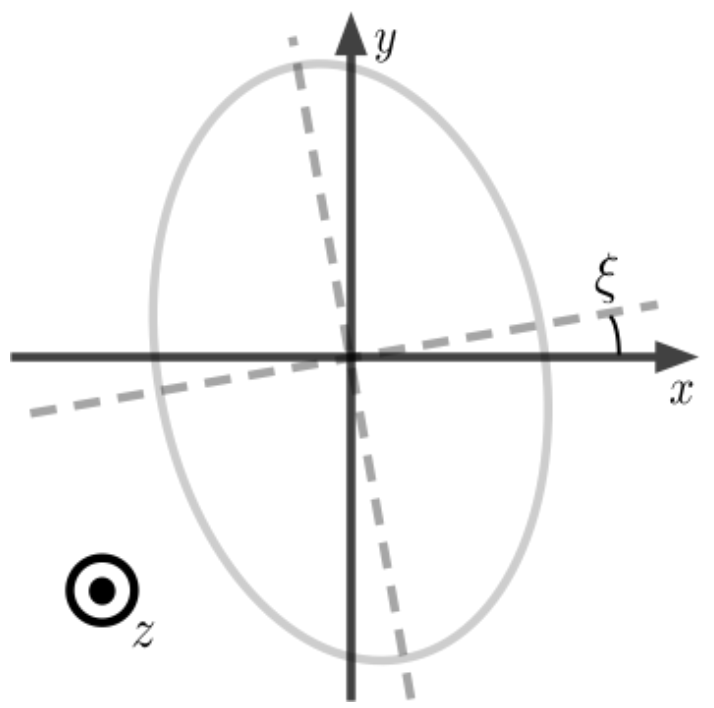

Figure 2.2: A body-fixed co-ordinate viewed from below. The dashed lines indicate the principal directions of curvature, rotated by $\xi$ from the principal axes of inertia ( $x-y$ axes).

\subsection{Small amplitude approximation of oscil- lations under $\omega_{z}=0$}

In this section, we consider the oscillation modes in the case of no spinning $\omega_{z}=0$ in the small amplitude approximation, namely, in the linear approximation in $\left|\omega_{x}\right|,\left|\omega_{y}\right| \ll \sqrt{g / a}$, which leads to $|x|,|y| \ll a,\left|u_{x}\right|,\left|u_{y}\right| \ll 1$, and $u_{z} \approx-1$.

In this regime, the $x$ and $y$ components of Eq. (2.10) can be linearized as

$$
\dot{\boldsymbol{u}}_{\perp} \approx \hat{\varepsilon} \boldsymbol{\omega}_{\perp}, \quad \hat{\varepsilon} \equiv\left(\begin{array}{cc}
0, & 1 \\
-1, & 0
\end{array}\right)=\hat{R}(-\pi / 2) .
$$

By using Eq. (2.8) with $u_{z} \approx-1$, Eq. (2.12) can be linearized as

$$
\begin{aligned}
\hat{J} \dot{\boldsymbol{\omega}}_{\perp} & \approx \frac{g}{a^{2}}(\boldsymbol{r} \times \boldsymbol{u})_{\perp} \\
& =-\frac{g}{a} \hat{\varepsilon}\left[-\hat{R}(\xi) \hat{\Theta}^{-1} \hat{R}^{-1}(\xi)+1\right] \boldsymbol{u}_{\perp},
\end{aligned}
$$

with the inertial matrix

$$
\hat{J} \equiv\left(\begin{array}{cc}
\alpha, & 0 \\
0, & \beta
\end{array}\right)
$$

From the linearized equations (2.18) and (2.19), we obtain

$$
\hat{J} \ddot{\boldsymbol{\omega}}_{\perp}=-\frac{g}{a}(\hat{\Gamma}-1) \boldsymbol{\omega}_{\perp}
$$


where

$$
\begin{aligned}
\hat{\Gamma} & \equiv \hat{R}(\xi+\pi / 2) \hat{\Theta}^{-1} \hat{R}^{-1}(\xi+\pi / 2) \\
& =\left(\begin{array}{ll}
\theta^{-1} \sin ^{2} \xi+\phi^{-1} \cos ^{2} \xi, & \left(\phi^{-1}-\theta^{-1}\right) \sin \xi \cos \xi \\
\left(\phi^{-1}-\theta^{-1}\right) \sin \xi \cos \xi, & \phi^{-1} \sin ^{2} \xi+\theta^{-1} \cos ^{2} \xi
\end{array}\right) .
\end{aligned}
$$

At this point, it is convenient to introduce the bra-ket notation for the row and column vector of $\boldsymbol{\omega}_{\perp}$ as $\left\langle\omega_{\perp}\right|$ and $\left|\omega_{\perp}\right\rangle$, respectively. With this notation, Eq. (2.21) can be put in the form of

$$
\left|\ddot{\tilde{\omega}}_{\perp}\right\rangle=-\hat{H}\left|\tilde{\omega}_{\perp}\right\rangle
$$

with

$$
\left|\tilde{\omega}_{\perp}\right\rangle \equiv \hat{J}^{1 / 2}\left|\omega_{\perp}\right\rangle
$$

and

$$
\begin{aligned}
\hat{H} & \equiv \frac{g}{a} \hat{J}^{-1 / 2}(\hat{\Gamma}-1) \hat{J}^{-1 / 2} \\
& =\frac{g}{a}\left(\begin{array}{cc}
\left(\Gamma_{11}-1\right) / \alpha, & \Gamma_{12} / \sqrt{\alpha \beta} \\
\Gamma_{12} / \sqrt{\alpha \beta}, & \left(\Gamma_{22}-1\right) / \beta
\end{array}\right),
\end{aligned}
$$

where $\Gamma_{i j}$ denotes the $i j$ component of $\hat{\Gamma}$. Note that $\hat{H}$ is symmetric.

The eigenvalue equation

$$
\hat{H}\left|\tilde{\omega}_{j}\right\rangle=\omega_{j}^{2}\left|\tilde{\omega}_{j}\right\rangle
$$

determines the two oscillation modes with $j=p$ or $r$, whose frequencies are given by

$$
\omega_{p, r}^{2}=\frac{1}{2}\left[\left(H_{11}+H_{22}\right) \pm \sqrt{\left(H_{11}-H_{22}\right)^{2}+4 H_{12}^{2}}\right]
$$

with

$$
\omega_{p} \geq \omega_{r}
$$

The orthogonal condition for $\left|\tilde{\omega}_{j}\right\rangle$ can be written using $\hat{\varepsilon}$ as

$$
\begin{array}{ll}
\left|\tilde{\omega}_{p}\right\rangle=\hat{\varepsilon}\left|\tilde{\omega}_{r}\right\rangle, & \left|\tilde{\omega}_{r}\right\rangle=-\hat{\varepsilon}\left|\tilde{\omega}_{p}\right\rangle, \\
\left\langle\tilde{\omega}_{r}\right|=\left\langle\tilde{\omega}_{p}\right| \hat{\varepsilon}, & \left\langle\tilde{\omega}_{p}\right|=-\left\langle\tilde{\omega}_{r}\right| \hat{\varepsilon} .
\end{array}
$$

In the case of zero skew angle, $\xi=0$, we have

$$
\begin{aligned}
& \omega_{p}^{2}=\left(\frac{g}{a}\right) \frac{1 / \phi-1}{\alpha} \equiv \omega_{p 0}^{2}, \\
& \omega_{r}^{2}=\left(\frac{g}{a}\right) \frac{1 / \theta-1}{\beta} \equiv \omega_{r 0}^{2},
\end{aligned}
$$


and the eigenvectors $\left|\omega_{p}\right\rangle$ and $\left|\omega_{r}\right\rangle$ are parallel to the $x$ and the $y$ axis, thus these modes correspond to the pitching and the rolling oscillations, respectively. This correspondence holds for $|\xi| \ll 1$ and $\omega_{p 0}>\omega_{r 0}$ as for a typical rattleback parameter, on which case we will discuss mostly in the following.

\subsection{Garcia and Hubbard's theory for the time for reversal}

Based on our formalism, it is quite straightforward to derive Garcia and Hubbard's formula for the reversal time of rotation.

\subsubsection{Asymmetric torque coefficients}

Due to the skewness, the pitching and the rolling are coupled with the spinning motion. We examine this coupling in the case of $\omega_{z}=0$ by estimating the averaged torques around the vertical axis caused by the pitching and rolling oscillations. From Eqs. (2.1) and (2.2) and the no-slip condition Eq. (2.3), the torque around $\boldsymbol{u}$ is given by

$$
T \equiv \boldsymbol{u} \cdot(\boldsymbol{r} \times \boldsymbol{F}) \approx-M a^{2}\left[\dot{\boldsymbol{\omega}}_{\perp} \cdot \hat{\varepsilon}(\hat{\Gamma}-1) \hat{\varepsilon} \boldsymbol{u}_{\perp}\right]
$$

within the linear approximation in $\boldsymbol{\omega}_{\perp}, \boldsymbol{u}_{\perp}$, and $\boldsymbol{r}_{\perp}$ discussed in the previous section.

We define the asymmetric torque coefficients $K_{p}$ and $K_{r}$ for each mode by

$$
-K_{p} \equiv \frac{\bar{T}_{p}}{\bar{E}_{p}}, \quad K_{r} \equiv \frac{\bar{T}_{r}}{\bar{E}_{r}}
$$

where $\bar{T}_{j}(j=p$ or $r)$ is the averaged torque over the oscillation period generated by each mode, and $\bar{E}_{j}$ is the corresponding averaged oscillation energy which can be estimated within the linear approximation as

$$
\bar{E} \approx M a^{2}\left(\alpha \overline{\omega_{x}^{2}}+\beta \overline{\omega_{y}^{2}}\right) .
$$

The minus sign for the definition of $K_{p}$ is inserted in order that both $K_{p}$ and $K_{r}$ should be positive as can be seen below. Note that the asymmetric torque coefficients are dimensionless. 
From Eqs. (2.33) and $(2.35),-K_{p}$ is given by

$$
\begin{aligned}
-K_{p} & =\frac{\left\langle\omega_{p}|\hat{\varepsilon}(\hat{\Gamma}-1) \hat{\varepsilon} \hat{\varepsilon}| \omega_{p}\right\rangle}{\left\langle\omega_{p}|\hat{J}| \omega_{p}\right\rangle} \\
& =-\frac{(a / g)\left\langle\tilde{\omega}_{p}\left|\hat{J}^{-1 / 2} \hat{\varepsilon} \hat{J}^{1 / 2} \hat{H}\right| \tilde{\omega}_{p}\right\rangle}{\left\langle\tilde{\omega}_{p} \mid \tilde{\omega}_{p}\right\rangle} \\
& =-\omega_{p}^{2} \frac{(a / g)\left\langle\tilde{\omega}_{p}\left|\hat{J}^{-1 / 2} \hat{\varepsilon} \hat{J}^{1 / 2}\right| \tilde{\omega}_{p}\right\rangle}{\left\langle\tilde{\omega}_{p} \mid \tilde{\omega}_{p}\right\rangle}
\end{aligned}
$$

with

$$
\hat{J}^{-1 / 2} \hat{\varepsilon} \hat{J}^{1 / 2}=\left(\begin{array}{cc}
0, & \sqrt{\beta / \alpha} \\
-\sqrt{\alpha / \beta}, & 0
\end{array}\right) .
$$

In the same way, $K_{r}$ is given by

$$
\begin{aligned}
K_{r} & =-\frac{(a / g)\left\langle\tilde{\omega}_{r}\left|\hat{J}^{-1 / 2} \hat{\varepsilon} \hat{J}^{1 / 2} \hat{H}\right| \tilde{\omega}_{r}\right\rangle}{\left\langle\tilde{\omega}_{r} \mid \tilde{\omega}_{r}\right\rangle} \\
& =\omega_{r}^{2} \frac{(a / g)\left\langle\tilde{\omega}_{p}\left|\hat{\varepsilon}\left(\hat{J}^{-1 / 2} \hat{\varepsilon} \hat{J}^{1 / 2}\right) \hat{\varepsilon}\right| \tilde{\omega}_{p}\right\rangle}{\left\langle\tilde{\omega}_{p} \mid \tilde{\omega}_{p}\right\rangle}
\end{aligned}
$$

with

$$
\hat{\varepsilon}\left(\hat{J}^{-1 / 2} \hat{\varepsilon} \hat{J}^{1 / 2}\right) \hat{\varepsilon}=\left(\begin{array}{cc}
0, & -\sqrt{\alpha / \beta} \\
\sqrt{\beta / \alpha}, & 0
\end{array}\right) .
$$

Eqs. (2.36)-(2.41) yield simple relations for $K_{p}$ and $K_{r}$ as

$$
\frac{K_{p}}{K_{r}}=\frac{\omega_{p}^{2}}{\omega_{r}^{2}}
$$

and

$$
\begin{aligned}
K_{p}-K_{r} & =\frac{(a / g)}{\left\langle\tilde{\omega}_{p} \mid \tilde{\omega}_{p}\right\rangle} \operatorname{Tr}\left[\hat{J}^{-1 / 2} \hat{\varepsilon} \hat{J}^{-1 / 2} \hat{H}\right] \\
& =-\frac{1}{2} \sin (2 \xi)\left(\frac{1}{\beta}-\frac{1}{\alpha}\right)\left(\frac{1}{\phi}-\frac{1}{\theta}\right) .
\end{aligned}
$$

Eqs. (2.42) and (2.43) are enough to determine

$$
\begin{aligned}
& K_{p}=-\frac{1}{2} \sin (2 \xi)\left(\frac{1}{\beta}-\frac{1}{\alpha}\right)\left(\frac{1}{\phi}-\frac{1}{\theta}\right) \frac{\omega_{p}^{2}}{\omega_{p}^{2}-\omega_{r}^{2}}, \\
& K_{r}=-\frac{1}{2} \sin (2 \xi)\left(\frac{1}{\beta}-\frac{1}{\alpha}\right)\left(\frac{1}{\phi}-\frac{1}{\theta}\right) \frac{\omega_{r}^{2}}{\omega_{p}^{2}-\omega_{r}^{2}} .
\end{aligned}
$$


Note that Eqs. (2.44) and (2.45) are consistent with the three requirements of rattlebacks: $\xi \neq 0, \alpha \neq \beta$, and $\theta \neq \phi$. Eqs. (2.44) and (2.45) are shown to be equivalent to the corresponding expressions Eq. (42a,b) in Garcia and Hubbard [16], although their expressions look quite involved. The proof is given in the appendix.

These results also show that

$$
K_{p} K_{r}>0 \text { and hence } \bar{T}_{p} \bar{T}_{r}<0,
$$

namely, the torques generated by the pitching and the rolling have always opposite signs to each other.

\subsubsection{Typical rattleback parameters}

Typical rattleback parameters fall in the region that satisfies the following two conditions: (i) the skew angle is small,

$$
|\xi| \ll 1,
$$

and (ii) the pitch frequency is higher than the roll frequency. Under these conditions, the modes $p$ and $r$ of Eq. (2.26) correspond to the pitching and the rolling oscillations respectively, and

$$
\omega_{p}^{2} \approx \omega_{p 0}^{2}, \quad \omega_{r}^{2} \approx \omega_{r 0}^{2}
$$

in accord with the inequality Eq. (2.28). From Eqs. (2.34), (2.44) and (2.45), the signs of the asymmetric torque coefficients and the averaged torques for typical rattlebacks are given by

$$
K_{p}>0 \text { and } K_{r}>0
$$

and

$$
\bar{T}_{p}<0 \text { and } \bar{T}_{r}>0
$$

by noting $\xi<0, \alpha>\beta, \theta>\phi$.

The fact that $\omega_{p 0}>\omega_{r 0}$ for a typical rattleback means that the shape factor, $1 / \phi-1$ or $1 / \theta-1$, contributes much more than the inertial factor, $1 / \alpha$ or $1 / \beta$, in Eqs. (2.31) and (2.32) although these two factors compete, i.e. $1 / \phi-1>1 / \theta-1$ and $1 / \alpha<1 / \beta$. This is a typical situation because the two curvatures of usual rattlebacks are markedly different, i.e., $\phi \ll \theta<1$ as can be seen in Fig. 1.1. Moreover, we can show that the pitch frequency is always higher than the roll frequency for an ellipsoid with a uniform mass density whose surface is given by $x^{2} / c^{2}+y^{2} / b^{2}+z^{2} / a^{2}=1\left(b^{2}>c^{2}>a^{2}\right)$. This also holds for a semi-ellipsoid for $b^{2}>c^{2}>(5 / 8) a^{2}$, where the co-ordinate system is the same as the ellipsoid. The proofs are given in the appendix. 


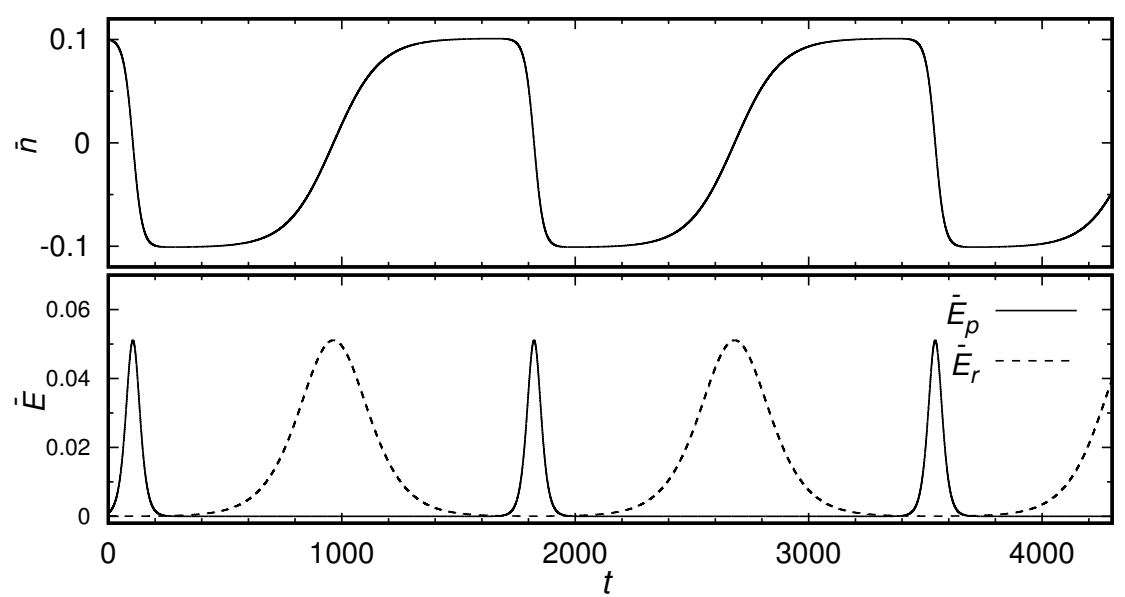

Figure 2.3: An example of the simplified dynamics Eqs. (2.52)-(2.54). $\bar{n}(0)=$ $0.1, \bar{E}_{p}(0)=10^{-3}, \bar{E}_{r}(0)=10^{-4}, K_{p}=0.5, K_{r}=0.1$, and $I_{\text {eff }}=10$.

\subsubsection{Time for reversal}

Now we study the time evolution of the spin $n$ defined as the vertical component of the angular velocity

$$
n \equiv \boldsymbol{u} \cdot \boldsymbol{\omega},
$$

assuming that the expressions for the asymmetric torque coefficients, $K_{p}$ and $K_{r}$, obtained above are valid even when $\omega_{z} \neq 0$. We consider the quantities $\bar{n}, \bar{E}_{p}$, and $\bar{E}_{r}$, averaged over the time scale much longer than the oscillation periods, yet much shorter than the time scale for spin change. Then, these averaged quantities should follow the evolution equations,

$$
\begin{aligned}
I_{\mathrm{eff}} \frac{d \bar{n}(t)}{d t} & =-K_{p} \bar{E}_{p}(t)+K_{r} \bar{E}_{r}(t), \\
\frac{d \bar{E}_{p}(t)}{d t} & =K_{p} \bar{n}(t) \bar{E}_{p}(t), \\
\frac{d \bar{E}_{r}(t)}{d t} & =-K_{r} \bar{n}(t) \bar{E}_{r}(t) .
\end{aligned}
$$

Here, $I_{\text {eff }}$ is the effective moment of inertia around $\boldsymbol{u}$ under the existence of the oscillations, and is assumed to be constant; it should be close to $I_{z z}$. Note that the dynamics shows chiral behavior for typical rattleback parameters because $K_{p} / K_{r}=\omega_{p}^{2} / \omega_{r}^{2} \gg 1$ with $K_{p}, K_{r}>0$ as argued in Sec. 2.3.2. 
As can be seen easily, the total energy $E_{\text {tot }}$ defined by

$$
E_{\mathrm{tot}} \equiv \frac{1}{2} I_{\mathrm{eff}} \bar{n}(t)^{2}+\bar{E}_{p}(t)+\bar{E}_{r}(t)
$$

is conserved. It can be seen that there is another invariant

$$
C \equiv \frac{1}{K_{p}} \ln \bar{E}_{p}+\frac{1}{K_{r}} \ln \bar{E}_{r},
$$

which has been discussed in connection with a Casimir invariant [15, 43]. With these two conservative quantities, general solutions of the three-variable system (2.52)-(2.54) should be periodic as shown in Fig. 2.3.

Let us consider the case where the spin is positive at $t=0$ and the sum of the oscillation energies are small compared to the spinning energy, i.e.

$$
\bar{n}(0) \equiv n_{i}>0, \quad \bar{E}_{p}(0)+\bar{E}_{r}(0) \ll \frac{1}{2} I_{\text {eff }} n_{i}^{2} .
$$

For a typical rattleback, the pitching develops and the rolling decays as long as $\bar{n}>0$ as can be seen from Eqs. (2.49), (2.53) and (2.54). Thus the rolling is irrelevant and can be ignored, i.e. $\bar{E}_{r}(t)=0$, to estimate the time for reversal. Then we can derive the equation

$$
\frac{d \bar{n}(t)}{d t}=-\frac{K_{p}}{2}\left(n_{0}^{2}-\bar{n}(t)^{2}\right)
$$

where the constant $n_{0}>0$ is defined by

$$
\frac{1}{2} I_{\mathrm{eff}} n_{0}^{2} \equiv E_{\mathrm{tot}}
$$

This can be easily solved as

$$
\bar{n}(t)=n_{0} \frac{\left(n_{0}+n_{i}\right) \exp \left(-n_{0} K_{p} t\right)-\left(n_{0}-n_{i}\right)}{\left(n_{0}+n_{i}\right) \exp \left(-n_{0} K_{p} t\right)+\left(n_{0}-n_{i}\right)}
$$

and we obtain the time for reversal $t_{r G H+}$ for the $n_{i}>0$ case as

$$
t_{r G H+}=\frac{1}{n_{0} K_{p}} \ln \left(\frac{n_{0}+n_{i}}{n_{0}-n_{i}}\right)
$$

by just setting $\bar{n}=0$ in Eq. (2.60).

Similarly, in the case of $n_{i}<0$, only the rolling develops and the pitching is irrelevant, thus we obtain $\bar{n}(t)$ and the time for reversal $t_{r G H-}$ as

$$
\bar{n}(t)=-n_{0} \frac{\left(n_{0}+\left|n_{i}\right|\right) \exp \left(-n_{0} K_{r} t\right)-\left(n_{0}-\left|n_{i}\right|\right)}{\left(n_{0}+\left|n_{i}\right|\right) \exp \left(-n_{0} K_{r} t\right)+\left(n_{0}-\left|n_{i}\right|\right)}
$$


and

$$
t_{r G H-}=\frac{1}{n_{0} K_{r}} \ln \left(\frac{n_{0}+\left|n_{i}\right|}{n_{0}-\left|n_{i}\right|}\right) .
$$

Eqs. (2.61) and (2.63) are Garcia-Hubbard formulae for the times for reversal [16].

\subsubsection{Parameter dependences of the time for reversal}

From the expressions of $K_{p}$ and $K_{r}$ given by Eqs. (2.44) and (2.45), we immediately notice that (i) the time for reversal is inversely proportional to the skew angle $\xi$ in the small skewness regime, and (ii) the ratio of the time for reversal $t_{r G H-} / t_{r G H+}$ is simply given by the squared ratio of the pitch frequency to the roll frequency $\omega_{p}^{2} / \omega_{r}^{2}$, provided initial values $n_{0}$ and $n_{i}$ are the same except their signs.

For a typical rattleback, $\omega_{p}^{2} \gg \omega_{r}^{2}$, thus $t_{r G H+} \ll t_{r G H-}$, i.e. the time for reversal is much shorter in the case of $n_{i}>0$ than in the case of $n_{i}<0$. Thus we call the spin direction of $n_{i}>0$ the unsteady direction [16], and that of $n_{i}<0$ the steady direction.

In the small skewness regime, this ratio of the squared frequencies is estimated as

$$
\frac{\omega_{p}^{2}}{\omega_{r}^{2}} \approx \frac{\omega_{p 0}^{2}}{\omega_{r 0}^{2}}=\frac{\beta}{\alpha} \frac{1 / \phi-1}{1 / \theta-1} .
$$

This becomes especially large as $\theta$ approaches 1 or as $\phi$ approaches 0 , namely, as the smaller radius of principal curvature approaches $a$, or as the larger radius of principal curvature becomes much larger than $a$. We remark that both of the inertial parameters $\alpha$ and $\beta$ are larger than 1 by definition Eq. (2.15), and cannot be arbitrarily large for a typical rattleback.

Let us consider these two limiting cases: $\phi \rightarrow 0$ and $\theta \rightarrow 1$ with $|\xi| \ll 1$. In the case of $\phi \rightarrow 0$,

$$
K_{p} \rightarrow \infty, \quad K_{r} \rightarrow(-\xi)\left(\frac{1}{\beta}-\frac{1}{\alpha}\right) \frac{\alpha}{\beta}\left(\frac{1}{\theta}-1\right),
$$

thus the time for reversal $t_{r G H}$ - remains constant while $t_{r G H+}$ approaches 0 . In the case of $\theta \rightarrow 1$,

$$
K_{p} \rightarrow(-\xi)\left(\frac{1}{\beta}-\frac{1}{\alpha}\right)\left(\frac{1}{\phi}-1\right), \quad K_{r} \rightarrow 0,
$$

thus $t_{r G H+}$ remains constant while $t_{r G H-}$ diverges to infinity, i.e. the negative spin rotation never reverses. 


\subsection{Three zones of the parameter space}

In this section, we briefly review Bondi's work [9], which applies to the small oscillation regime yet the spin $n$ is not necessary small.

There always exists a steady solution of the equations of motion Eqs. (2.10) and (2.12),

$$
\boldsymbol{\omega}(0)=(0,0, \text { const. })^{t} \text { and } \boldsymbol{u}(0)=(0,0,-1)^{t},
$$

namely, constant vertical spinning at the bottom $\boldsymbol{r}=(0,0, a)^{t}$. Since the spin $n$ changes in much slower times scale than the pitching and the rolling, he assumed it as a constant when considering the instability of the oscillations. By examining the roots of the characteristic equation in the linear stability analysis of the oscillations under the constant spin, Bondi classified the six-dimensional parameter space $(\alpha, \beta, \gamma, \theta, \phi, \xi)$ into 3 "zones". We call them Zone 0, Zone I and Zone II after Bondi (Zone 0 was named by Garcia and Hubbard [16]). These zones are classified using following two auxiliary variables $\mu_{B}$ and $\kappa_{B}$ :

$$
\begin{aligned}
\mu_{B}=2- & (\theta+\phi)-(\alpha+\beta-\gamma)(\theta+\phi-2 \theta \phi), \\
\kappa_{B}=1- & \frac{1}{2}(\alpha+\beta-2 \gamma)(\theta+\phi) \\
& \quad+(\alpha-\gamma)(\beta-\gamma) \theta \phi-\frac{1}{2}(\alpha-\beta)(\theta-\phi) \cos (2 \xi) .
\end{aligned}
$$

It can be shown that $\kappa_{B}>\mu_{B}$.

We call the subspace where $\mu_{B}>0$ Zone 0 . In this zone, either the pitching or the rolling grows for arbitrary $n$. We call the subspace where $\mu_{B}<0$ and $\kappa_{B}>0$ Zone I. In this zone, there exists the threshold spin value $n_{c 1}$ such that the spin reversal due to rolling does not occur where $|n|>\left|n_{c 1}\right|$. In this case, the motion asymptotically approaches vertical steady spinning, i.e., the steady solution is linearly stable. If $|n|<\left|n_{c 1}\right|$, the behavior is the same as Zone 0 rattleback, i.e., either the pitching or the rolling is unstable depending on a spinning direction. Lastly, the subspace where $\mu_{B}<0$ and $\kappa_{B}<0$ is called Zone II. In this zone, the second threshold $n_{c 2}$ can be defined. While Bondi did not show the asymptotic motion which corresponds to $|n|>\left|n_{c 2}\right|$, Gracia and Hubbard numerically showed that unlike Zone I motion, the body-fixed $z$-axis forms a certain angle to the vertical vector $\boldsymbol{u}$ asymptotically. For $\left|n_{c 1}\right|<|n|<\left|n_{c 2}\right|$, the motion asymptotically approaches vertical steady spinning motion as in Zone I, and for $|n|<\left|n_{c 1}\right|$, either the pitching or the rolling is unstable as in Zone 0 . 
The expressions of $n_{c 1}$ and $n_{c 2}$ are given by

$$
\begin{aligned}
& n_{c 1}=-\left(\frac{g}{a} \frac{-\nu_{B}}{\mu_{B}}\right)^{1 / 2} \\
& n_{c 2}=-\left(\frac{g}{a}\right)^{1 / 2}\left(\frac{2 \nu_{B}}{\left.\left[\left(\lambda_{B}+\mu_{B}\right)^{2}-4 \nu_{B} \kappa_{B}\right]^{1 / 2}-\left(\lambda_{B}+\mu_{B}\right)\right]}\right)^{1 / 2}
\end{aligned}
$$

where

$$
\begin{aligned}
\lambda_{B} & =\frac{1}{2}(\alpha+\beta)(\theta+\phi-2 \theta \phi)-\frac{1}{2}(\alpha-\beta)(\theta-\phi) \cos (2 \xi), \\
\nu_{B} & =(1-\theta)(1-\phi) .
\end{aligned}
$$

Note that $n_{c 1}$ does not depend on $\xi$.

Linderg and Longman [18] performed simulations for a rattleback with a parameter set which corresponds to Zone I with $\left|n_{c 1}\right|=31.7 / \mathrm{sec}$. They observed instability for both spinning directions when $\left|n_{i}\right|=1 / \mathrm{sec}$, which is consistent with the Bondi's theory. In the next chapter we investigate the simulation results of Zone 0 and Zone I rattlebacks with the initial spin $\left|n_{i}\right|$ larger than $\left|n_{c 1}\right|$.

Bondi has shown that Zone II does not exist if

$$
I_{x x}<I_{z z}, \quad \text { or equivalently }(\alpha-1)-\gamma<0 .
$$

For an ellipsoid $x^{2} / c^{2}+y^{2} / b^{2}+z^{2} / a^{2}=1\left(b^{2}>c^{2}>a^{2}\right)$ with a uniform mass density,

$$
\alpha-\gamma=\frac{1}{5}\left[6-\left(\frac{c}{a}\right)^{2}\right]<1
$$

thus Zone II does not exist. This holds for a semi-ellipsoid as shown in the appendix. In addition to that, a simple rattleback model we introduce in the next chapter also can not have Zone II. Detailed analysis of the Zone II rattleback is beyond the scope of this thesis.

\subsection{Oscillations with a small constant spin}

In this section we review Case and Jalal [11], which shows that either the pitching or the rolling grows depending on the spinning direction when we start with a small constant spin. This corresponds to a special case of Bondi's work. 
We consider small deviations $\left|u_{x}\right|,\left|u_{y}\right| \ll 1,\left|\omega_{x}\right|,\left|\omega_{y}\right| \ll \sqrt{g / a}$ around the steady solution Eq. (2.67) under the small constant spin $|n| \ll \sqrt{g / a}$, and examine the linear stability of this solution, i.e., $\boldsymbol{\omega}(t)=(0,0,-n)^{t}$ and $\boldsymbol{u}(t)=(0,0,-1)^{t}$.

First, Eq. (2.18) is linearized in the presence of the small spin as

$$
\dot{\boldsymbol{u}}_{\perp} \approx \hat{\varepsilon}\left(\boldsymbol{\omega}_{\perp}-n \boldsymbol{u}_{\perp}\right) .
$$

Next we linearize Eq. (2.12). To this end, we linearize Eqs. (2.8) using $\hat{\Gamma}$ defined in Eq. (2.22) as

$$
\frac{\boldsymbol{r}_{\perp}}{a} \approx \hat{\varepsilon} \hat{\Gamma} \hat{\varepsilon} \boldsymbol{u}_{\perp}=\left(\begin{array}{cc}
-\Gamma_{22}, & \Gamma_{12} \\
\Gamma_{12}, & -\Gamma_{11}
\end{array}\right) \boldsymbol{u}_{\perp}
$$

with $z \approx a$. Note that $\Gamma_{12}=\Gamma_{21}$ because $\hat{\Gamma}$ is symmetric. Then, $(\boldsymbol{r} \times \boldsymbol{u})_{\perp}$ can be written using $\hat{\Gamma}$ as,

$$
(\boldsymbol{r} \times \boldsymbol{u})_{\perp} \approx a \hat{S} \boldsymbol{u}_{\perp}, \quad \hat{S}=\left(\begin{array}{cc}
-\Gamma_{12}, & \Gamma_{11}-1 \\
-\Gamma_{22}+1, & \Gamma_{12}
\end{array}\right) .
$$

In addition to the terms in the case of $\omega_{z}=0$, following terms are added to the linearized equation of Eq. (2.12):

$$
\begin{aligned}
& {[\boldsymbol{\omega} \times(\hat{I} \boldsymbol{\omega})]_{\perp} \approx n\left(\begin{array}{c}
\left(-I_{z}+I_{y y}\right) \omega_{y} \\
\left(-I_{x}+I_{z}\right) \omega_{x}
\end{array}\right),} \\
& {[\dot{\boldsymbol{r}} \cdot(\boldsymbol{r} \cdot \boldsymbol{\omega})]_{\perp} \approx-n a^{2}\left(\begin{array}{cc}
-\Gamma_{22}, & \Gamma_{12} \\
\Gamma_{12}, & -\Gamma_{11}
\end{array}\right) \dot{\boldsymbol{u}}_{\perp}}
\end{aligned}
$$

and

$$
(\boldsymbol{r} \times \boldsymbol{\omega})(\boldsymbol{r} \cdot \boldsymbol{\omega}) \approx-n a^{2}\left(-\omega_{y}, \omega_{x}, 0\right)^{t} .
$$

By collecting the terms of Eq. (2.12) with non-zero components, we obtain

$$
\hat{I} \dot{\boldsymbol{\omega}}+M r^{2} \dot{\boldsymbol{\omega}}=-\boldsymbol{\omega} \times(\hat{I} \boldsymbol{\omega})+M[\dot{\boldsymbol{r}}(\boldsymbol{r} \cdot \boldsymbol{\omega})-(\boldsymbol{r} \times \boldsymbol{\omega})(\boldsymbol{r} \cdot \boldsymbol{\omega})+g \boldsymbol{r} \times \boldsymbol{u}] .
$$

After some manipulations, its $x$ and $y$ components become

$$
\alpha \dot{\omega}_{x}=n(\gamma-\beta) \omega_{y}+n\left(\Gamma_{22} \dot{u}_{x}-\Gamma_{12} \dot{u}_{y}\right)+\frac{g}{a}\left[-\Gamma_{12} u_{x}+\left(\Gamma_{11}-1\right) u_{y}\right]
$$

and

$$
\beta \dot{\omega}_{y}=n(\alpha-\gamma) \omega_{x}+n\left(-\Gamma_{12} \dot{u}_{x}+\Gamma_{11} \dot{u}_{y}\right)+\frac{g}{a}\left[-\left(\Gamma_{22}-1\right) u_{x}+\Gamma_{12} u_{y}\right]
$$




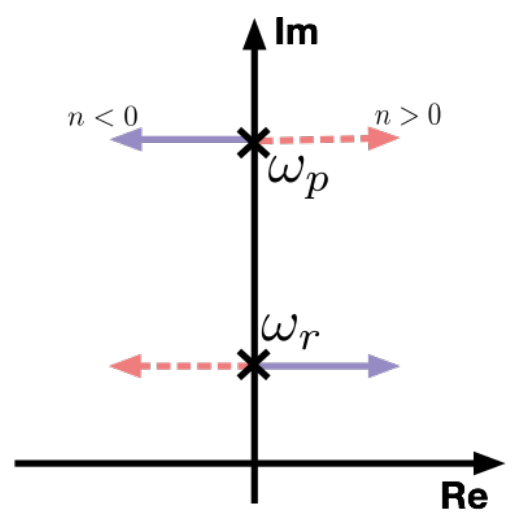

Figure 2.4: A schematic illustration of the loci of the roots when the spin $n$ departs slightly from 0 .

Eqs. (2.83) and (2.84) can be written in the matrix form as

$$
\hat{J} \dot{\boldsymbol{\omega}}_{\perp}=n \hat{X}_{0} \boldsymbol{\omega}_{\perp}+n \hat{X}_{1} \dot{\boldsymbol{u}}_{\perp}+\frac{g}{a} \hat{S} \boldsymbol{u}_{\perp}
$$

where

$$
\hat{X}_{0}=\left(\begin{array}{cc}
0, & (\gamma-\beta) \\
(\alpha-\gamma), & 0
\end{array}\right), \quad \hat{X}_{1}=\left(\begin{array}{cc}
\Gamma_{22}, & -\Gamma_{12} \\
-\Gamma_{12}, & \Gamma_{11}
\end{array}\right) .
$$

With the notation $\delta \equiv g / a$, Eqs. (2.76) and (2.85) lead to

$$
\begin{aligned}
\hat{J} \dot{\boldsymbol{\omega}}_{\perp} & =n \hat{X}_{0} \boldsymbol{\omega}_{\perp}+n \hat{X}_{1} \hat{\varepsilon}\left(\boldsymbol{\omega}_{\perp}-n \boldsymbol{u}_{\perp}\right)+\delta \hat{S} \boldsymbol{u}_{\perp} \\
& \approx n\left(\hat{X}_{0}+\hat{X}_{1} \hat{\varepsilon}\right) \boldsymbol{\omega}_{\perp}+\delta \hat{S} \boldsymbol{u}_{\perp},
\end{aligned}
$$

then the differential equations for $\boldsymbol{\omega}_{\perp}$ and $\boldsymbol{u}_{\perp}$ can be put in the form of

$$
\left(\begin{array}{c}
\dot{\boldsymbol{\omega}}_{\perp} \\
\dot{\boldsymbol{u}}_{\perp}
\end{array}\right)=\hat{A}\left(\begin{array}{c}
\boldsymbol{\omega}_{\perp} \\
\boldsymbol{u}_{\perp}
\end{array}\right)
$$

where

$$
\hat{A}=\left(\begin{array}{cccc}
n \frac{\Gamma_{12}}{\alpha}, & \frac{n}{\alpha}\left(\Gamma_{22}+(\gamma-\beta)\right), & -\delta \frac{\Gamma_{12}}{\alpha}, & \frac{\delta}{\alpha}\left(\Gamma_{11}-1\right) \\
\frac{n}{\beta}\left(-\Gamma_{11}+(\alpha-\gamma)\right), & n \frac{-\Gamma_{12}}{\beta}, & -\frac{\delta}{\beta}\left(\Gamma_{22}-1\right), & \delta \frac{\Gamma_{12}}{\beta} \\
0, & 1, & 0, & -n \\
-1, & 0, & n, & 0
\end{array}\right) .
$$


Its characteristic equation $\left|\hat{A}-\lambda_{c} \hat{I}_{4}\right|=0$ is given by

$$
\begin{aligned}
\lambda_{c}^{4}+\lambda_{c}^{3} n \Gamma_{12}\left(\frac{1}{\beta}-\frac{1}{\alpha}\right)+\lambda_{c}^{2}[ & \left.\frac{\delta}{\alpha}\left(\Gamma_{11}-1\right)+\frac{\delta}{\beta}\left(\Gamma_{22}-1\right)\right] \\
& +\frac{\delta^{2}}{\alpha \beta}\left[\left(\Gamma_{11}-1\right)\left(\Gamma_{22}-1\right)-\Gamma_{12}^{2}\right]=0 .
\end{aligned}
$$

Here we have dropped $O\left(n^{2}\right)$ terms. Note that the first order term in $\lambda_{c}$ vanishes. When $n=0$, the eigenvalues become $\pm i \omega_{p}, \pm i \omega_{r}$. In the presence of the small third order term, the roots depart from the imaginary axis (Fig. 2.4). The characteristic equation can be rearranged as

$$
\left(\lambda_{c}^{2}+\omega_{p}^{2}\right)\left(\lambda_{c}^{2}+\omega_{r}^{2}\right)=-\lambda_{c}^{3} n \Gamma_{12}(1 / \beta-1 / \alpha) .
$$

By substituting $\lambda_{c}=i \omega_{p}+\sigma_{p}, i \omega_{r}+\sigma_{r}$ into the equation and collecting the lowest order terms in $\sigma_{p}$ and $\sigma_{r}$, we obtain

$$
\sigma_{p}=-\frac{n \Gamma_{12}}{2} \frac{(1 / \beta-1 / \alpha) \omega_{p}^{2}}{\left(\omega_{p}^{2}-\omega_{r}^{2}\right)} \quad \sigma_{r}=\frac{n \Gamma_{12}}{2} \frac{(1 / \beta-1 / \alpha) \omega_{r}^{2}}{\left(\omega_{p}^{2}-\omega_{r}^{2}\right)} .
$$

Eq. (2.92) is equivalent to that by Case and Jalal [11]. Note that $\Gamma_{12}<0$ since $\xi<0$. For typical rattlebacks with $\omega_{p} \approx \omega_{p 0}>\omega_{r 0} \approx \omega_{r}$, the pitching develops if $n>0$, while the rolling develops if $n<0$; this is consistent with our theory.

\subsection{Comparison of our theory with related works}

The simplified dynamics in Section 2.3 can be compared with some related works. We have reviewed Case and Jalal's calculation of the growth rates [11] in the previous section. Their results can be expressed as

$$
\sigma_{p}=\frac{n}{2} K_{p}, \quad \sigma_{r}=-\frac{n}{2} K_{r} .
$$

The factor $1 / 2$ comes from the choice of the variables; they chose the contact point co-ordinates, while we choose the oscillation energies, which are second order quantities of their variables.

Moffatt and Tokieda [15] obtained equations for the oscillation amplitudes of pitching and rolling, $P$ and $R$, and the spinning $S$ as

$$
\frac{d}{d \tau}\left(\begin{array}{c}
P \\
R \\
S
\end{array}\right)=\left(\begin{array}{c}
R \\
\lambda P \\
0
\end{array}\right) \times\left(\begin{array}{c}
P \\
R \\
S
\end{array}\right)=\left(\begin{array}{c}
\lambda P S \\
-R S \\
R^{2}-\lambda P^{2}
\end{array}\right),
$$


where $\tau$ is rescaled time, and $\lambda$ is the squared ratio of the pitch frequency to the roll frequency. Their assumptions are that (i) the rattleback is an oblong, mass-uniform ellipsoidal one with a small skew angle, and (ii) the spin and the oscillations are small.

By changing variables of our simplified dynamics Eqs. (2.52)-(2.54) as

$$
\bar{E}_{p}=\frac{P_{s}^{2}}{2}, \quad \bar{E}_{r}=\frac{R_{s}^{2}}{2}, \quad \bar{n}=S_{s}
$$

we obtain

$$
\dot{P}_{s}=\frac{K_{p}}{2} P_{s} S_{s}, \quad \dot{R}_{s}=-\frac{K_{r}}{2} R_{s} S_{s}, \quad I_{\text {eff }} \dot{S}_{s}=\frac{K_{r}}{2} R_{s}^{2}-\frac{K_{p}}{2} P_{s}^{2},
$$

which are equivalent to Eq. (2.94) because $K_{p} / K_{r}=\lambda$.

The mathematical structure of Eq. (2.94) was investigated recently in more detail by Yoshida et al. [43]. They found that Eq. (2.94) can be reformulated as

$$
\dot{\boldsymbol{X}}=\{\boldsymbol{X}, H\} \text {, where } \boldsymbol{X} \equiv(P, R, S)^{t} \text { and } H \equiv \frac{1}{2}\left(P^{2}+R^{2}+S^{2}\right) .
$$

Here $\{A, B\}$ is the Poisson bracket defined as

$$
\{A, B\} \equiv\left(\partial_{i} A\right) J_{i j}\left(\partial_{j} B\right), \quad \text { with } \quad \hat{J}=\left(\begin{array}{ccc}
0, & 0, & \lambda P \\
0, & 0, & -R \\
-\lambda P, & R, & 0
\end{array}\right) .
$$

Such a system is called Lie-Poisson system [43], and is known to have an invariant called Casimir invariant $C_{I}$, which satisfies

$$
\left(\partial_{i} C_{I}\right) J_{i j}=0
$$

In their case, this invariant is given by

$$
C_{I}=P R^{\lambda}
$$

$C_{I}$ corresponds to the invariant $C$ in Eq. (2.56), because

$$
2 e^{\left(C K_{p}\right) / 2}=P_{s} R_{s}^{\lambda} .
$$




\section{Chapter 3}

\section{Simulation}

In this chapter, we present numerical simulations of the equations of motion and compare them with Garcia-Hubbard formulae Eqs. (2.61) and (2.63) reformulated in the previous chapter.

\subsection{Shell-dumbbell model}

To consider a rattleback whose inertial and geometrical parameters can be set separately, we construct a simple model of the rattleback, or the shelldumbbell model, which consists of a light shell and two dumbbells: the light shell defines the shape of the lower part of the rattleback and the dumbbells represent the masses and the moments of inertia. The shell is a paraboloid given by Eq. (2.4). The dumbbells consist of couples of weights, $m_{x} / 2$ and $m_{y} / 2$, fixed at $\left( \pm r_{x}, 0,0\right)$ and $\left(0, \pm r_{y}, 0\right)$ in the body-fixed co-ordinate, respectively (Fig. 3.1). Then the total mass is

$$
M=m_{x}+m_{y}
$$

and the inertia tensor is diagonal with its principal moments

$$
\begin{aligned}
I_{x x} & =m_{y} r_{y}^{2}, \quad I_{y y}=m_{x} r_{x}^{2}, \\
I_{z z} & =m_{y} r_{y}^{2}+m_{x} r_{x}^{2} .
\end{aligned}
$$

Note that the simple relation

$$
I_{z z}=I_{x x}+I_{y y}
$$

holds for the shell-dumbbell model. We define

$$
f_{s d} \equiv I_{y y} / I_{z z}
$$




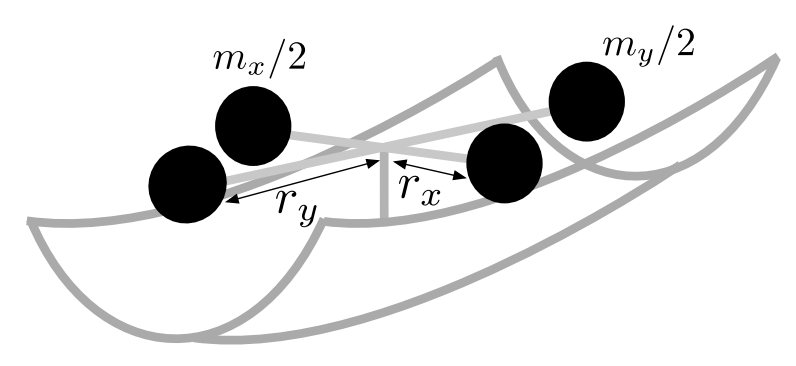

Figure 3.1: A schematic illustration of the shell-dumbbell model.

then the dimensionless parameters $\alpha, \beta$, and $\gamma$ defined by Eq. (2.15) are given by,

$$
\gamma=I_{z z} / M a^{2}, \alpha=\left(1-f_{s d}\right) \gamma+1, \beta=f_{s d} \gamma+1 .
$$

The parameter $f_{s d}$ satisfies $0<f_{s d}<0.5$, since we have assumed $\alpha>\beta$.

As stated in the previous chapter, Zone II does not exist if $(\alpha-1)-\gamma<0$ (Eq. (2.74)). As for the shell-dumbbell model,

$$
(\alpha-1)-\gamma=-f_{s d} \gamma<0
$$

It satisfies the relation (2.74), thus there is no Zone II rattleback for the shell-dumbbell model.

The shell-dumbbell model makes it easier to visualize an actual object represented by the model with a set of parameters, and is used in the following simulations for determining the parameter ranges.

\subsection{Simulation methods}

The equations of motion (2.10) and (2.12) with the contact point conditions (2.4) and (2.8) are numerically integrated by the fourth-order Runge-Kutta method with an initial condition $\boldsymbol{\omega}(0)$ and $\boldsymbol{u}(0)$. In the simulations, we take

$$
\boldsymbol{u}(0)=(0,0,-1)^{t}
$$

and specify $\boldsymbol{\omega}(0)$ as

$$
\boldsymbol{\omega}(0)=\left(\left|\omega_{x y 0}\right| \cos \psi,\left|\omega_{x y 0}\right| \sin \psi,-n_{i}\right)
$$

in terms of $\left|\omega_{x y 0}\right|, \psi$, and $n_{i}$. According to the simplified dynamics Eqs. (2.52)(2.54), the irrelevant mode of oscillation does not affect the dynamics sensitively as long as the relevant mode exists and the initial spin energy is much 
Table 3.1: Two sets of parameters used in the simulations: GH used by Garcia and Hubbard [16] and SD for the present shell-dumbbell model. For $\mathrm{SD}$, the parameter values are chosen randomly from the ranges shown in the table, and averages and/or distributions of simulation results are presented.

\begin{tabular}{ccccccc}
\hline & $\gamma$ & $f_{s d}$ & $\alpha, \beta$ & $\theta$ & $\phi$ & $-\xi(\mathrm{deg})$ \\
\hline $\mathrm{GH}$ & 12.28 & - & $13.04,1.522$ & 0.6429 & 0.0360 & 1.72 \\
$\mathrm{SD}$ & {$[5,15]$} & {$[0.05,0.15]$} & - & {$[0.6,0.95]$} & {$[0.01,0.1]$} & $(0,6]$ \\
\hline
\end{tabular}

larger than the initial oscillation energy. Thus we choose $|\omega(0)\rangle=\left(\omega_{x 0}, \omega_{y 0}\right)^{t}$ in the direction of the relevant eigenmode, i.e.

$$
\psi=\psi_{p} \text { for } n_{i}>0, \text { and } \psi=\psi_{r} \text { for } n_{i}<0,
$$

where $\psi_{p}$ and $\psi_{r}$ are the angles of the eigenvectors $\left|\omega_{p}\right\rangle$ and $\left|\omega_{r}\right\rangle$ from the $x$-axis, respectively.

Numerical results are presented in the unit system where $M, a$, and

$$
\tilde{t} \equiv 1 / \tilde{\omega} \equiv \sqrt{a / g}
$$

as units of mass, length, and time. The size of the time step for the numerical integration is taken to be $0.002 \tilde{t}$. In numerics, we determine the time for reversal $t_{r}$ by the time at which $n=\boldsymbol{\omega} \cdot \boldsymbol{u}$ becomes zero for the first time, and they are compared with Garcia-Hubbard formulae (2.61) and (2.63); $n_{0}$ is determined as

$$
\frac{\gamma n_{0}^{2}}{2}=\frac{1}{2}\left(\alpha \omega_{x 0}^{2}+\beta \omega_{y 0}^{2}+\gamma \omega_{z 0}^{2}\right),
$$

assuming $I_{\mathrm{eff}}=I_{z z}$ at $t=0$. Here the potential energy $U(\boldsymbol{u})$ is set to zero where $\boldsymbol{u}(0)=(0,0,-1)^{t}$.

The parameters used in the simulations are listed in Table 3.1. For the parameter set SD, the ranges are shown. When numerical results are plotted against $K_{p}$ or $K_{r}$, given by Eqs. (2.44) or (2.45) respectively, sets of parameters are chosen randomly from the ranges until resulting $K_{p}$ or $K_{r}$ falls within the range of $\pm 0.1 \%$ of a target value. The ranges of SD are chosen to meet the following two conditions: (i) $0<\phi \ll \theta<1, \beta<\alpha$, and $|\xi| \ll 1$ and (ii) the pitch frequency should be higher than the roll frequency. As argued in Section 2.3, usual rattlebacks such as one in Fig. 1.1 satisfy these two conditions. Fig. 3.2 shows the cumulative distributions for the eigenfrequencies $\omega_{p}$ and $\omega_{r}$, and their approximate expressions $\omega_{p 0}$ and $\omega_{r 0}$ for the parameter set SD; it shows $\left(\omega_{p} / \omega_{r}\right)>1.3$ in accordance with the condition (ii).

The parameter set $\mathrm{GH}$ gives $K_{p}=0.553$ and $K_{r}=0.0967$, and the distributions of $K_{p}$ and $K_{r}$ for SD are shown in Fig. 3.3, where one can see 


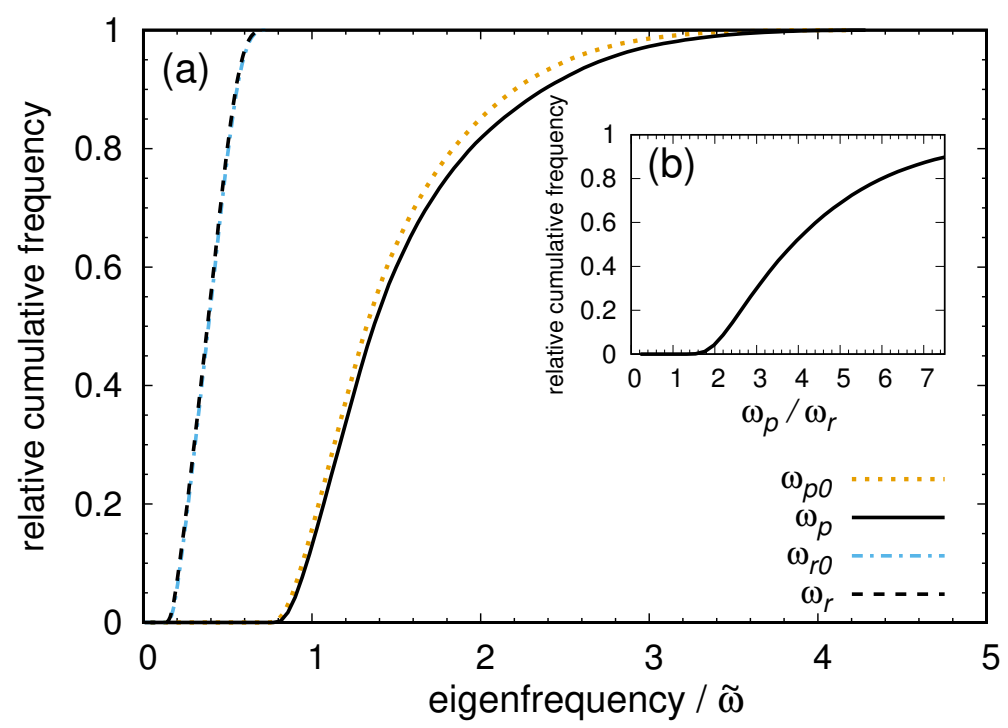

Figure 3.2: (a) Cumulative distributions of the pitch and the roll frequencies for the parameter set SD in Table 3.1; $\omega_{p}$ and $\omega_{r}$ of Eq. (2.26) and their 0-th order approximation $\omega_{p 0}$ and $\omega_{r 0}$ by Eqs. (2.31) and (2.32) are shown. The inset shows the cumulative distribution of $\omega_{p} / \omega_{r}$. The number of samples is $10^{6}$.

$K_{p} \gg K_{r}$. From Eq. (2.42), this corresponds to $\omega_{p}^{2} \gg \omega_{r}^{2}$, i.e., the pitch frequency is significantly faster than the roll frequency. Consequently, the time for reversal is much shorter for the unsteady direction $n_{i}>0$, where the pitching is induced, than for the steady direction $n_{i}<0$, where the rolling is induced. We denote the time for reversal for the unsteady direction as $t_{r u}$ and that for the steady direction as $t_{r s}$ when we consider a specific spinning direction.

\subsection{Simulation results for the parameter set GH}

\subsubsection{General behavior for the parameter set GH}

In Fig. 3.4 we show a typical simulation result of the time evolution of the spin $n(t)$ along with the angular velocities $\omega_{x}(t)$ and $\omega_{y}(t)$ for the parameter set GH (Table 3.1) in the case of the unsteady direction $n_{i}>0$ (a), and the steady direction $n_{i}<0(\mathrm{~b})$.

Fig. 3.4(a-1) shows that the spin $n$ changes its sign from positive to negative at $t_{r u} \approx 112 \tilde{t}$, and Fig. $3.4(\mathrm{~b}-1)$ shows the spin $n$ changes its sign from 


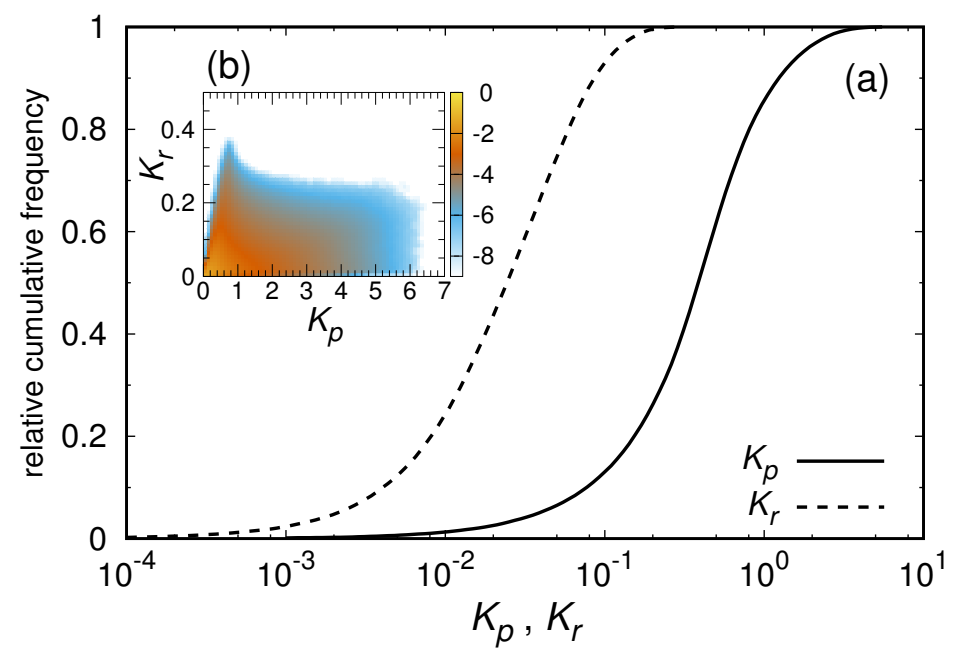

Figure 3.3: (a) Cumulative distributions of the asymmetric torque coefficients $K_{p}$ and $K_{r}$ for SD (Table 3.1). The number of samples is $10^{5}$. (b) A 2-d color plot for the distribution of $\left(K_{p}, K_{r}\right)$. The color code shown is in the logarithmic scale for the relative frequency $P\left(K_{p}, K_{r}\right)$, i.e., $-9 \leq \log _{10} P\left(K_{p}, K_{r}\right) \leq 0$. The number of samples is $10^{8}$.

negative to positive at $t_{r s} \approx 810 \tilde{t}$. Garcia and Hubbard's solution $\bar{n}(t)$ of Eqs. (2.60) and (2.62) are shown by the dashed lines in Fig. 3.4(a-1) and (b$1)$, respectively; they are in good agreement with the numerical simulations.

The angular velocities $\omega_{x}$ and $\omega_{y}$ oscillate in much shorter time scale, and their amplitudes evolve differently depending on the spin direction. In the case of Fig. 3.4(a), where the positive initial spin reverses to negative, the amplitude of $\omega_{x}$ becomes large and reaches its maximum around $t_{r u}$; the amplitude of $\omega_{y}$ also becomes large around both sides of $t_{r u}$ but shows the local minimum at $t_{r u}$. Both of $\omega_{x}$ and $\omega_{y}$ oscillate at the pitch frequency $\omega_{p} \approx 1.44 \tilde{\omega}$. In the case of Fig. 3.4(b) where the negative spin reverses to positive, the situation is similar but the amplitude of $\omega_{y}$ reaches its maximum around $t_{r s}$, and $\omega_{x}$ and $\omega_{y}$ oscillate at the roll frequency $\omega_{r} \approx 0.602 \tilde{\omega}$.

These features can be understood based on the analysis in the previous chapter as follows. The positive spin induces the pitching, which is mainly represented by $\omega_{x}$ because eigenvector of the pitching $\left|\omega_{p}\right\rangle$ are nearly parallel to the $x$-axis, i.e., $\psi_{p} \approx-17^{\circ}$. Likewise, the negative spin induces the rolling, mainly represented by $\omega_{y}$ because $\psi_{r} \approx 88^{\circ}$. The local minima of the amplitude for $\omega_{y}$ in Fig. 3.4(a-3), or $\omega_{x}$ in Fig. 3.4(b-2), at the times for reversal are tricky; it might mean that the eigenvector of the pitching (rolling) deviates 


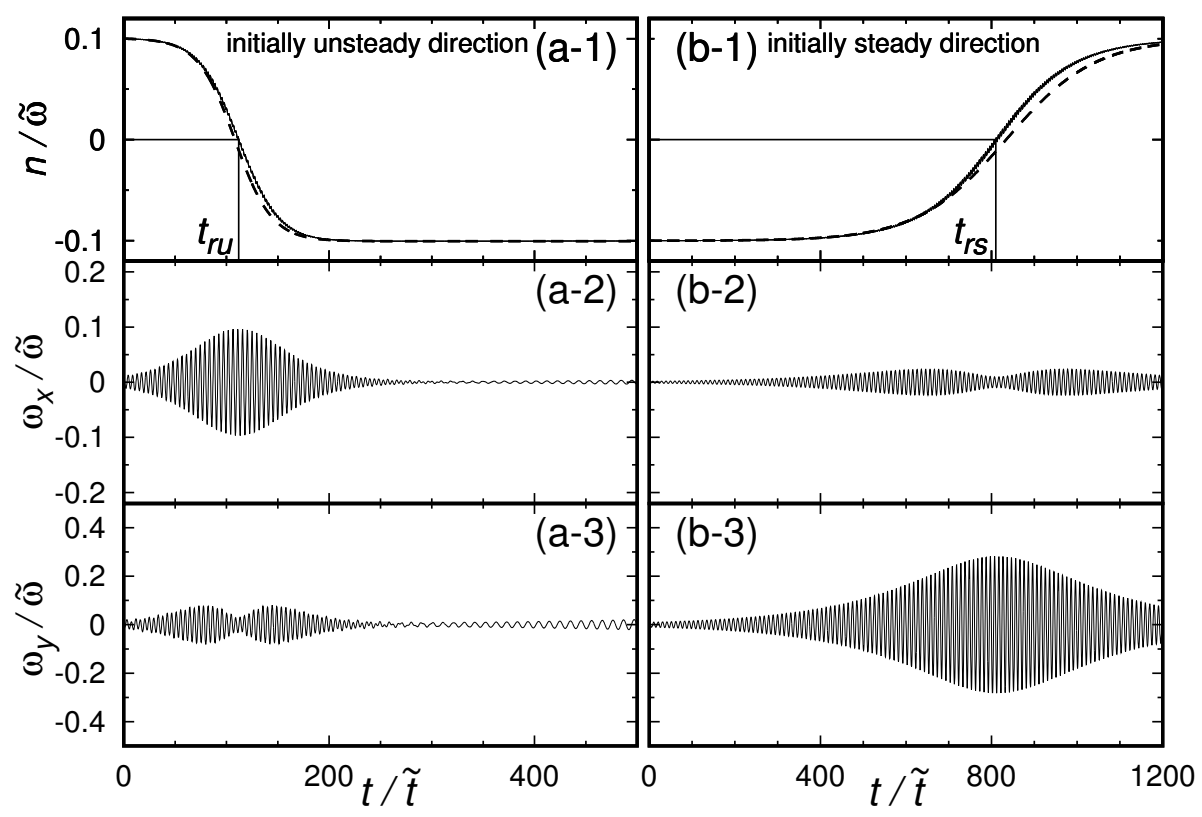

Figure 3.4: A typical spin evolution and the corresponding $\omega_{x}$ and $\omega_{y}$ for GH (Table 3.1). (a) The case of the initial spin in the unsteady direction. The initial condition is specified by Eqs. (3.8)-(3.10) with $n_{i}=0.1 \tilde{\omega}$ and $\left|\omega_{x y 0}\right|=0.01 \tilde{\omega}$. (b) The case of the initial spin in the steady direction with $n_{i}=-0.1 \tilde{\omega}$ and $\left|\omega_{x y 0}\right|=0.01 \tilde{\omega}$. The dashed lines in (a-1) and (b-1) show Garcia and Hubbard's solution $\bar{n}(t)$ given by Eqs. (2.60) and (2.62), respectively.

more from the $x$-axis ( $y$-axis) for $\omega_{z} \neq 0$ than that for $\omega_{z}=0$, as a result, the pitching (rolling) mode has larger projection on the $y$-axis ( $x$-axis) for $\omega_{z} \neq 0$.

Note that for given $\left|n_{i}\right|$, the maximum value of $\omega_{y}$ in Fig. 3.4(b-3) is larger than that of $\omega_{x}$ in (a-2). This is due to $\alpha \gg \beta$; the oscillation energy around zero spin for the both cases should be the same, which gives $\alpha \overline{\omega_{x}^{2}} \approx \beta \overline{\omega_{y}^{2}}$ thus $\sqrt{\overline{\omega_{x}^{2}}}<\sqrt{\overline{\omega_{y}^{2}}}$

\subsubsection{Comparison with Garcia-Hubbard formulae}

Garcia and Hubbard [16] simulated the equations of motion for several $n_{0}$ between $0.021 \tilde{\omega}$ and $0.13 \tilde{\omega}$ (6 data points for the unsteady direction and 4 for the steady direction) with fixed $n_{i}^{2} / n_{0}^{2}$ (0.95 for the unsteady direction, 0.75 for the steady direction) and obtained good agreement with $t_{r G H \pm}$. In this subsection, we present similar simulations but for wider range of $n_{i}$ and 
$\left|\omega_{x y 0}\right|$.

The times for reversal $t_{r u}$ and $t_{r s}$ as functions of initial spin $n_{i}$ are shown in Fig. 3.5. The simulation data is fitted well to $t_{r G H+}$ for the unsteady direction where $n_{i}$ is small, but $t_{r u}$ deviates drastically from $t_{r G H+}$ beyond $n_{i} \simeq 0.3 \tilde{\omega}$ for $\left|\omega_{x y 0}\right|=0.001 \tilde{\omega}$ and beyond $n_{i} \simeq 0.1 \tilde{\omega}$ for $\left|\omega_{x y 0}\right|=0.1 \tilde{\omega}$. As $\left|\omega_{x y 0}\right|$ becomes larger, the spin value at which $t_{r u}$ starts to deviate from $t_{r G H+}$ becomes smaller. As for the $n_{i}$ dependence, $t_{r u}$ starts to increase around $n_{i} \approx 0.4 \tilde{\omega}$ and appears to converge to the value $30 \tilde{t}$ irrespective of $\left|\omega_{x y 0}\right|$. Fig. 3.5(c) shows a spin evolution with $n_{i}=0.6 \tilde{\omega}$. The spin oscillates widely at the pitch frequency, and the spin behavior is qualitatively different from typical behaviors at small $n_{i}$ and from Garcia and Hubbard's solution $\bar{n}(t)$ of Eq. (2.60).

For the steady direction, the simulation data is fitted well to $t_{r G H-}$ for small $\left|n_{i}\right|$, but when $\left|n_{i}\right| \gtrsim 0.6 \tilde{\omega}$, the rattleback does not reverse its spin and wobbles "chaotically" between $n_{i}<n(t)<0$, as shown in Fig. 3.5(d) for the case $n_{i}=-0.7 \tilde{\omega}$.

As we have argued in the previous chapter, there always exists a steady solution, $\boldsymbol{\omega}(0)=(0,0 \text {, const. })^{t}$ and $\boldsymbol{u}(0)=(0,0,-1)^{t}$, and Bondi [9] has shown that for the steady direction, this is linearly stable for $n<n_{c 1}<0$, where $n_{c 1}(<0)$ is given by

$$
n_{c 1}^{2} \equiv \frac{g}{a} \frac{-(1-\theta)(1-\phi)}{2-(\theta+\phi)-(\alpha+\beta-\gamma)(\theta+\phi-2 \theta \phi)} .
$$

$\left|n_{c 1}\right|$ of the parameter set GH is $1.68 \tilde{\omega}$. As we have pointed out above, the spin does not reverse at $n_{i}=0.7 \tilde{\omega}$ already. However, this does not contradict with Bondi's theory, because the spin actually deviates from the steady solution.

For larger $n_{i}, t_{r G H+}$ tends to underestimate $t_{r u}$, as already noted by Garcia and Hubbard [16] for the parameter set GH. This tendency can be also seen in Fig. 3.4(a-1), where one can see that Garcia and Hubbard's solution $\bar{n}(t)$ of Eq. (2.60) changes its sign earlier than the simulation. On the other hand, $t_{r G H-}$ tends to overestimate $t_{r s}$, in contrast to the case of the unsteady direction, where $t_{r G H+}$ underestimates $t_{r u}$. This has also been noted by Garcia and Hubbard for the parameter set GH, and can be seen by Garcia and Hubbard's solution $\bar{n}(t)$ in Fig. 3.4(b-1).

\subsection{Simulation with the parameter set SD}

We present detailed results of the simulations for the ranges of the parameters given by SD in Table 3.1. 

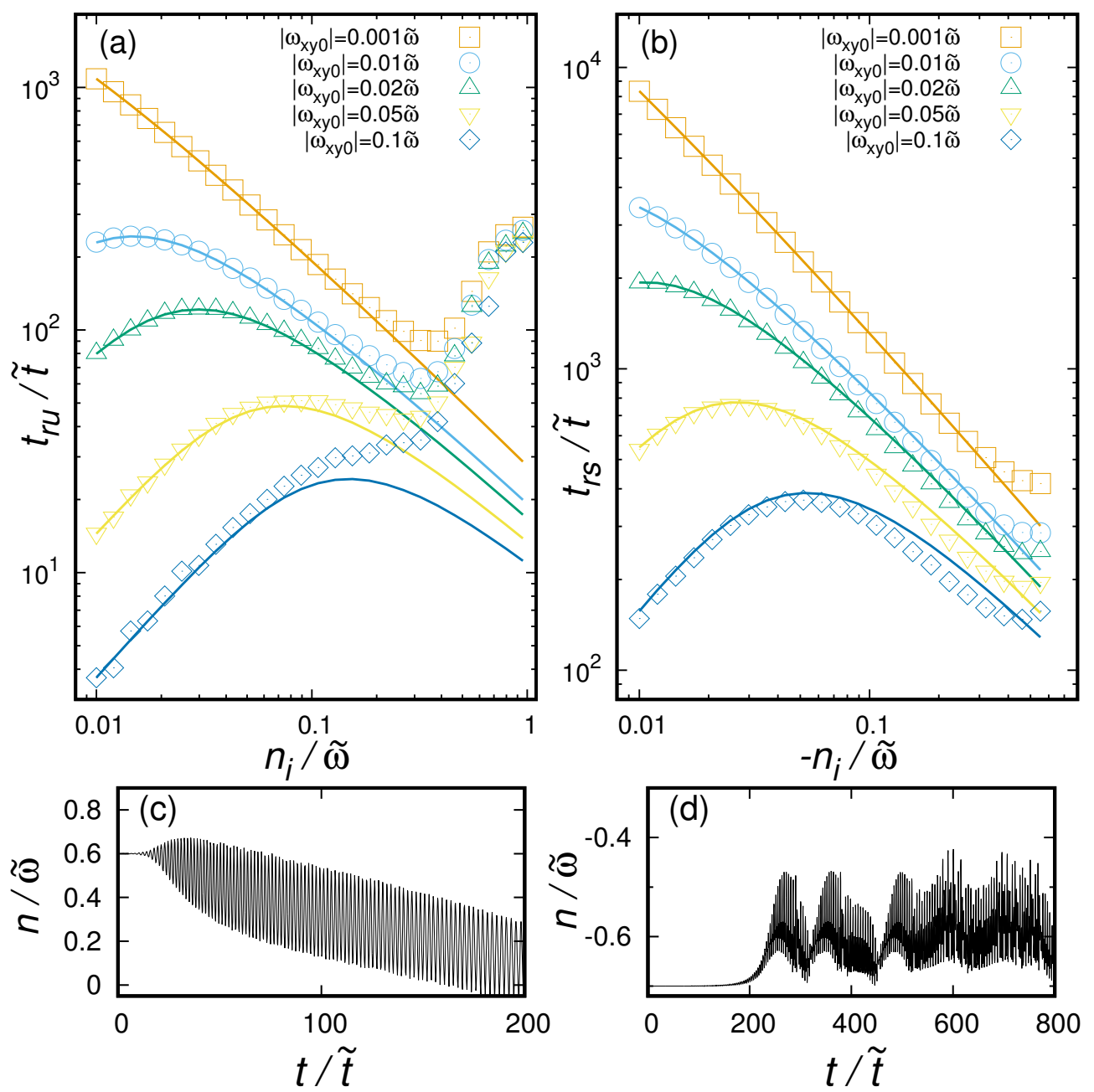

Figure 3.5: Times for reversal $t_{r u}$ and $t_{r s}$ (marks) as functions of initial spin $n_{i}$ for GH (Table 3.1), along with the Garcia-Hubbard formula $t_{r G H \pm}$ given by Eqs. (2.61) and (2.63) (solid lines). (a) The case of the initial spin in the unsteady direction $\left(n_{i}>0\right)$. (b) The case of the initial spin in the steady direction $\left(n_{i}<0\right)$. (c) A spin evolution with high unsteady initial spin $n_{i}=0.6 \tilde{\omega}$. $\left|\omega_{x y 0}\right|=0.01 \tilde{\omega}$. (d) A spin evolution with high steady initial spin $n_{i}=-0.7 \tilde{\omega} \cdot\left|\omega_{x y 0}\right|=0.01 \tilde{\omega}$. 


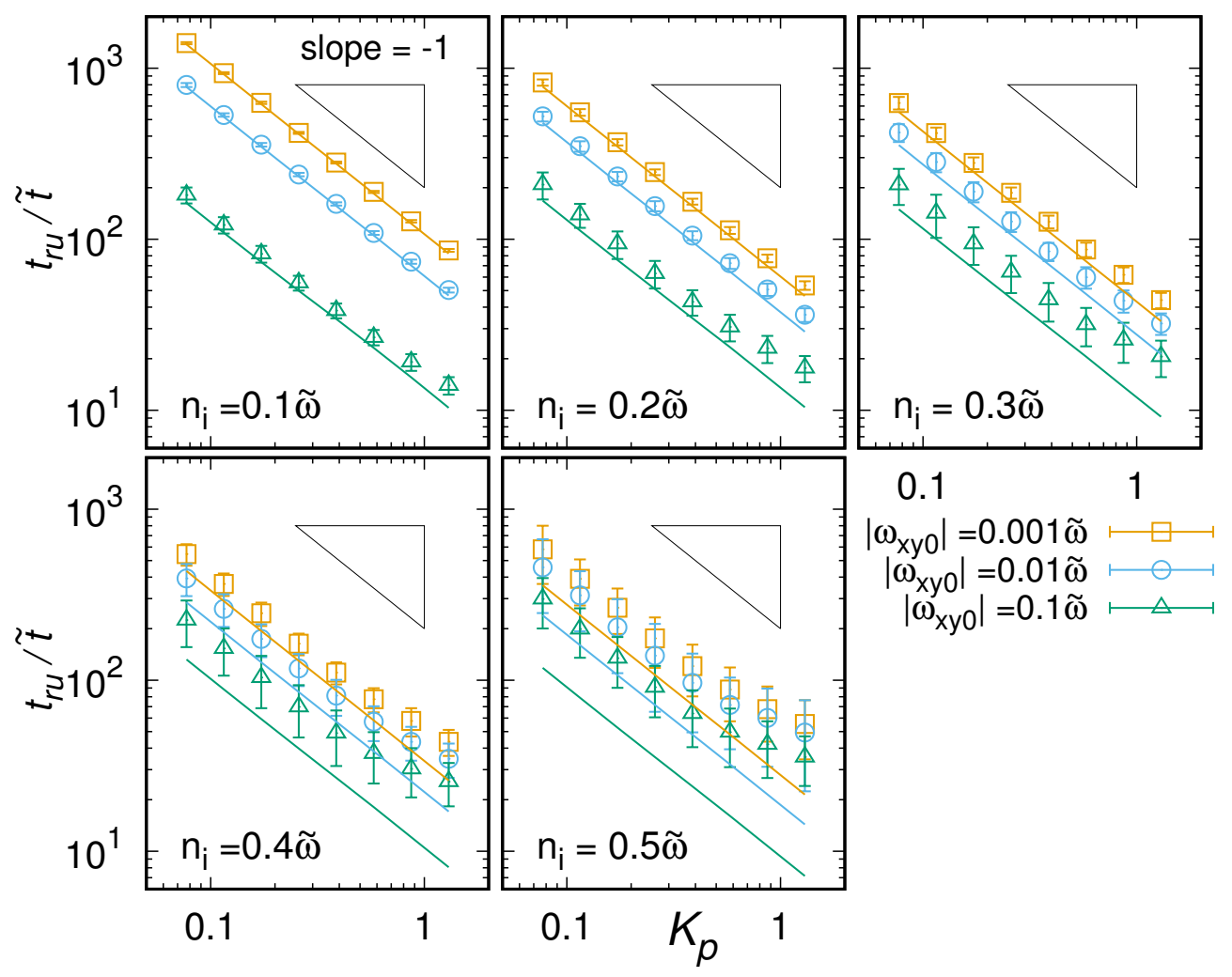

Figure 3.6: Time for reversal of the unsteady direction $t_{r u}$ for the parameter set SD (Table 3.1) as a function of the asymmetric torque coefficient $K_{p}$ in the logarithmic scale. The error bars indicate one standard deviation of 1000 samples for each data point. The solid lines are $t_{r G H+}$ given by Eq. (2.61), calculated using the mean values of $n_{0}$. 


\subsubsection{Unsteady initial spin direction $\left(n_{i}>0\right)$}

In this case, the system behaves basically as we expect from Garcia-Hubbard formula unless the initial spin or oscillation is too large. Fig. 3.6 shows the time for reversal $t_{r u}$ as a function of $K_{p}$ when spun in the unsteady direction. The results are plotted against $K_{p}$ by the procedure described in Sec. 3.2.

When the initial spin $n_{i}$ is $n_{i} \lesssim 0.2 \tilde{\omega}$ with $\left|\omega_{x y 0}\right|=0.001 \tilde{\omega}, 0.01 \tilde{\omega}, t_{r u}$ is in good agreement with Garcia-Hubbard formula $t_{r G H+}$ of Eq. (2.61), i.e. almost inversely proportional to $K_{p}$ with small scatter around the average. For a given $n_{i}$, as the initial oscillation amplitude $\left|\omega_{x y 0}\right|$ becomes large, the standard deviations of $t_{r u}$ become large, and the average of $t_{r u}$ deviates upward from the Garcia-Hubbard formula $t_{r G H+}$, which is derived with the small amplitude approximation of $\omega_{x}$ and $\omega_{y}$. For larger $n_{i}, t_{r G H+}$ also underestimates $t_{r u}$, as in the case of GH.

For $n_{i} \gtrsim 0.4 \tilde{\omega}, t_{r u}$ deviates notably upward from Garcia-Hubbard formula $t_{r G H+}$. As $n_{i}$ increases, the average of $t_{r u}$ increases and the standard deviations become large. The spin oscillates widely at the pitch frequency as in the case of $\mathrm{GH}$, which is qualitatively different from typical spin behaviors at small $n_{i}$ and from Garcia and Hubbard's solution $\bar{n}(t)$ of Eq. (2.60) as in Fig. 3.4(a-1). In this region, Garcia-Hubbard formula is no longer valid.

\subsubsection{Steady initial spin direction $\left(n_{i}<0\right)$}

Much more complicated phenomena are observed when spun in the steady direction. When the initial spin $\left|n_{i}\right|$ is small enough, the spin simply reverses as shown in Fig. 3.4(b-1). We call this simple reversal behavior Type R. For larger $\left|n_{i}\right|$, however, there appear some cases where the spin never reverses; in such cases there are two types of behaviors: steady spinning at $n_{s s}$ (Type SS), and spin wobbling around $n_{w}\left(n_{s s}<n_{w}<0\right.$, Type SW). For Type SS samples, $n_{s s}$ is slightly less than $n_{i}$, i.e. $n_{s s}<n_{i}<0$, because small initial rolling decays and its energy is converted to the spin energy. Typical spin evolutions of a Type SS sample and a Type SW sample are shown in Fig. 3.7(b-1) and (b-2).

Fig. 3.7(a) shows the $K_{r}$ dependence of the fractions of Types R, SS, and $\mathrm{SW}$ for various initial conditions given by $n_{i}$ and $\left|\omega_{x y 0}\right|$. For each sample, we wait up to $t=5 t_{r H_{-}}$; the spin evolution is classified as Type $\mathrm{R}$ if it reverses. If it does not, the spin evolution is classified as Type SS if the initial rolling amplitude decays monotonously, and classified as Type SW if the spin $n$ starts wobbling by the time $5 t_{r G H_{-}}$. The other samples, in which the rolling grows slowly yet shows no visible spin change by the time $5 t_{r G H-}$, are labeled "unclassified" in Fig. 3.7. Such samples may show spin reversal or 


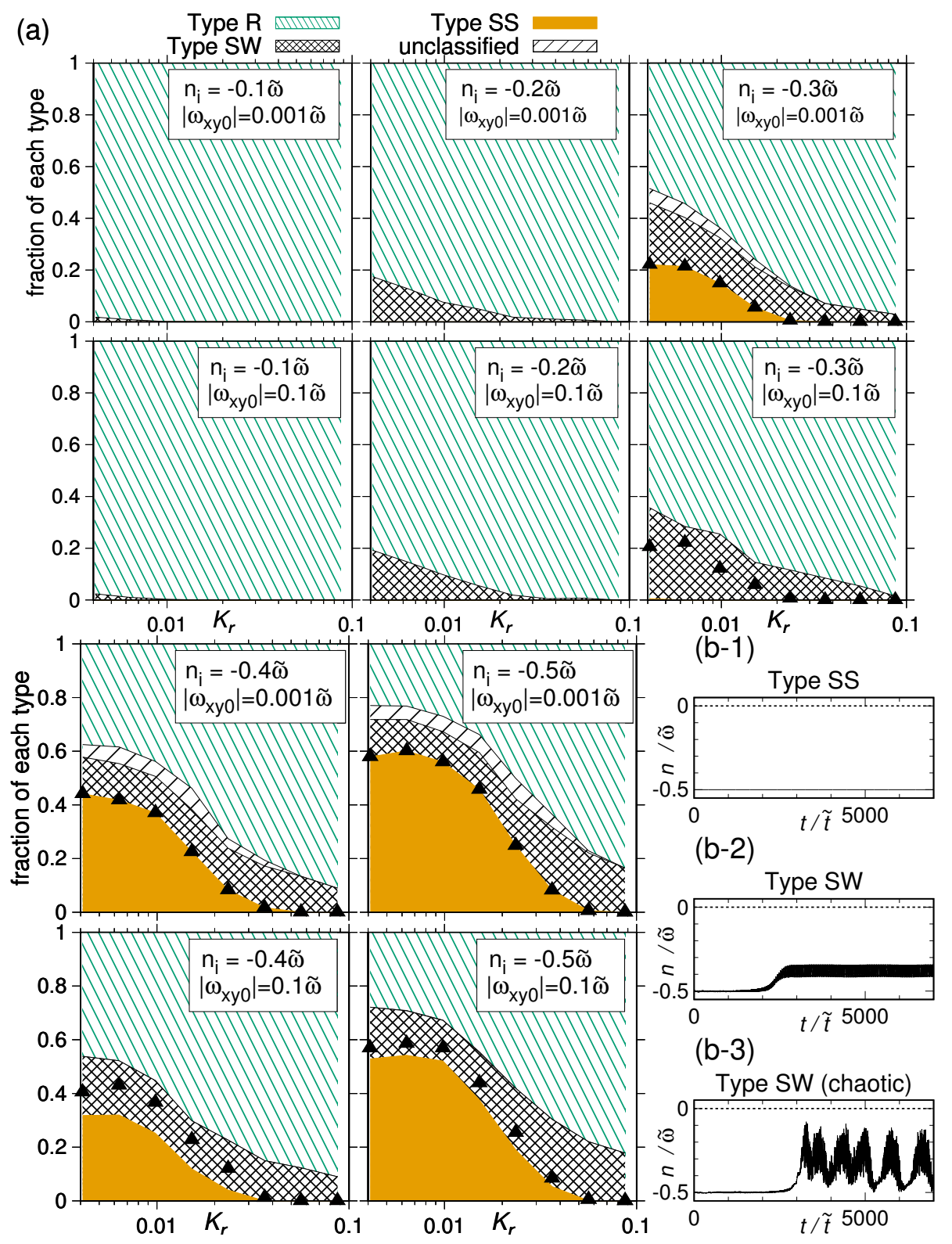

Figure 3.7: (a) Fractions of Types R, SS, and SW for the steady direction for 8 values of $K_{r}$ with various initial conditions $\left|\omega_{x y 0}\right|$ and $n_{i}$. Parameters are randomly chosen from SD (Table 3.1). The number of the samples is 1000 for each $K_{r}$. Filled triangles show the fractions of samples whose $\left|n_{c 1}\right|$ is smaller than $\left|n_{i}\right|$. (b) Typical spin evolutions of a Type SS sample and a Type SW sample, along with an example of "chaotic" oscillation found for $K_{r}=0.0041$ with $n_{i}=-0.5 \tilde{\omega}$. 
spin wobbling if we take much longer simulation time. Type SS appears for $\left|n_{i}\right| \gtrsim 0.3 \tilde{\omega}$ and its fraction increases as $\left|n_{i}\right|$ increases. The fraction is larger for smaller $K_{r}$ and smaller $\left|\omega_{x y 0}\right|$, i.e., $\left|\omega_{x y 0}\right|=0.001 \tilde{\omega}$. Type SW appears for $\left|n_{i}\right| \gtrsim 0.1 \tilde{\omega}$ and its fraction is also larger for smaller $K_{r}$, but stays around 0.2 for $\left|n_{i}\right| \gtrsim 0.4 \tilde{\omega}$.

Fig. 3.8 shows the $K_{r}$ dependence of $t_{r s}$ only for the samples of Type R, which shows a spin reversal behavior. For small $\left|n_{i}\right| \lesssim 0.2 \tilde{\omega}$ with $\left|\omega_{x y 0}\right|=$ $0.01 \tilde{\omega}, 0.001 \tilde{\omega}, t_{r s}$ is in good agreement with Garcia-Hubbard formula $t_{r G H-}$ of Eq. (2.63), and the average of $t_{r u}$ is almost inversely proportional to $K_{r}$. As in the case of the unsteady direction, the standard deviations of $t_{r s}$ become large and the average of $t_{r s}$ deviates downward from $t_{r G H-}$ as initial oscilla-

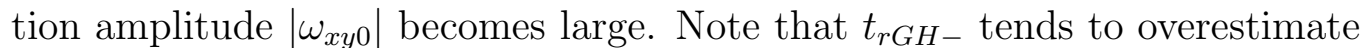
$t_{r s}$, in contrast to the case of the unsteady direction, where $t_{r G H+}$ underestimates $t_{r u}$ as we have shown for the parameter set GH. For $\left|n_{i}\right| \gtrsim 0.3 \tilde{\omega}$, one may notice the standard deviations are large for $K_{r} \ll 0.1$. In these cases, we find that some samples appear to spin stably for quite a long time, i.e., several times of $t_{r G H_{-}}$, and then abruptly starts to reverse its sign. During the time period $t<t_{r s}$, the rolling grows much more slowly than it should as predicted by the theory in Section 2.3. Such samples make both the average and standard deviation large as shown in Fig. 3.8.

Next we consider the Type SS (steady spinning) samples. In our case of the parameter set $\mathrm{SD}, n_{c 1}(<0)$ is given by

$$
n_{c 1}^{2}=\frac{g}{a} \frac{1}{(1-\phi)^{-1}+(1-\theta)^{-1}-4} .
$$

Note that $\alpha+\beta-\gamma=2$ in our case of the shell-dumbbell model. We recall that when the denominator of Eq. (3.14) is negative, such a threshold does not actually exit, and the steady solution is always unstable. Note also that $n_{c 1}$ does not depend on $\xi$. In Fig. 3.9 we show Zone 0 and Zone I of the parameter space projected in the $\theta-\phi$ plane. As we have shown in Section 3.1, there is no Zone II rattleback for the present model. For our choice of parameters where $\phi \ll \theta$, most rattlebacks are in Zone I.

In Fig. 3.7, filled triangles show the fraction of samples whose $\left|n_{c 1}\right|$ is smaller than $\left|n_{i}\right|$, which should correspond with the ratio of Type SS. For $\left|\omega_{x y 0}\right|=0.001 \tilde{\omega}$, all samples whose $\left|n_{c 1}\right|$ is smaller than $\left|n_{i}\right|$ actually show Type SS behaviors and vice versa. On the other hand, for $\left|\omega_{x y 0}\right|=0.1 \tilde{\omega}$, there are some samples whose $\left|n_{c 1}\right|$ is smaller than $\left|n_{i}\right|$ yet do not show Type SS behavior; for $n_{i}=-0.3 \tilde{\omega}$, there are only several Type SS samples out of 8000 samples, which cannot be seen in Fig. 3.7(a), and for $\left|n_{i}\right| \gtrsim 0.4 \tilde{\omega}$, the fractions of Type SS for $\left|\omega_{x y 0}\right|=0.1 \tilde{\omega}$ are smaller than those for $\left|\omega_{x y 0}\right|=$ 


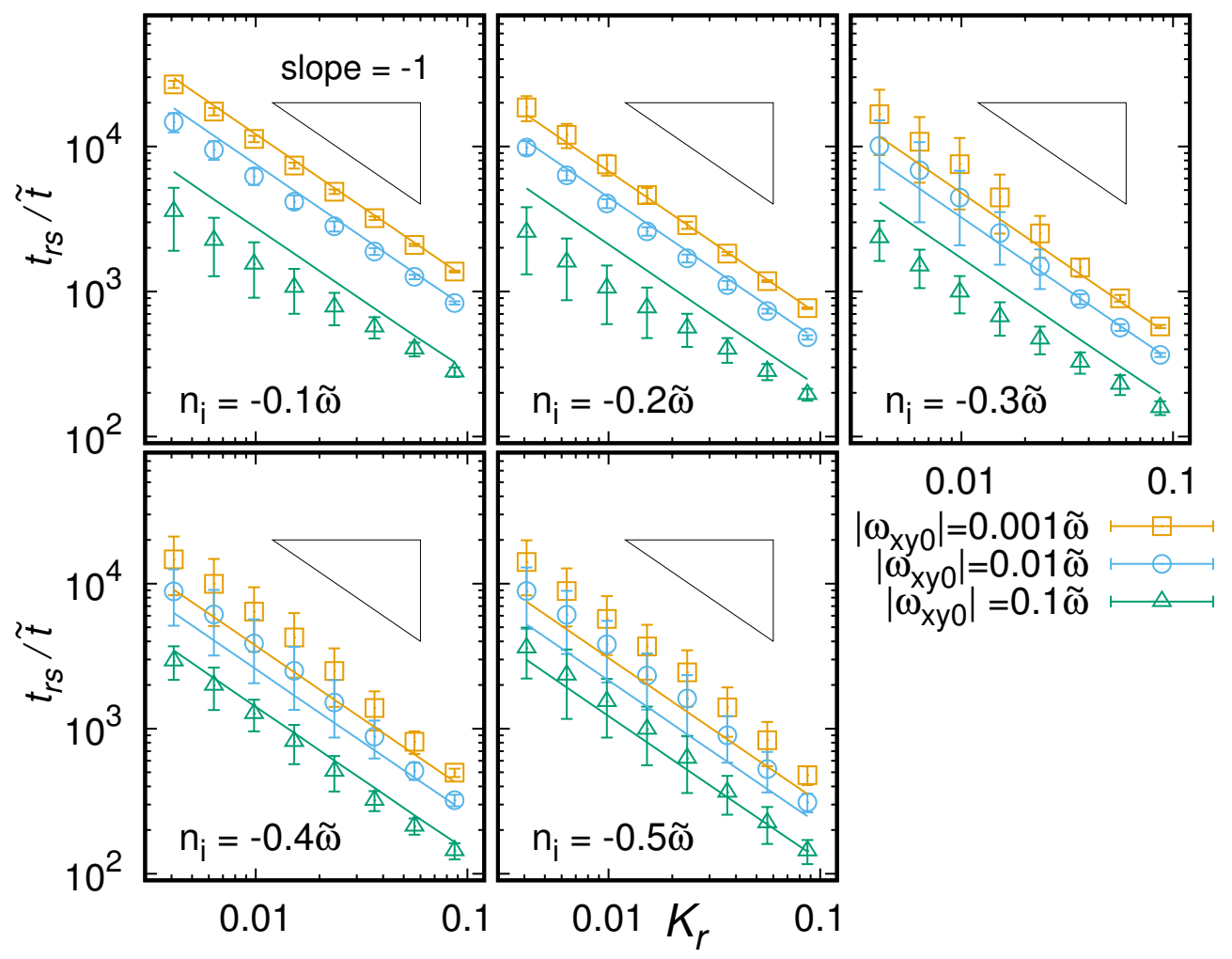

Figure 3.8: Time for reversal $t_{r s}$ for the steady direction as a function of $K_{r}$ in the logarithmic scale. Each data point represents the average with the standard deviation of Type $\mathrm{R}$ samples out of 1000 simulations from the parameter set SD (Table 3.1). 


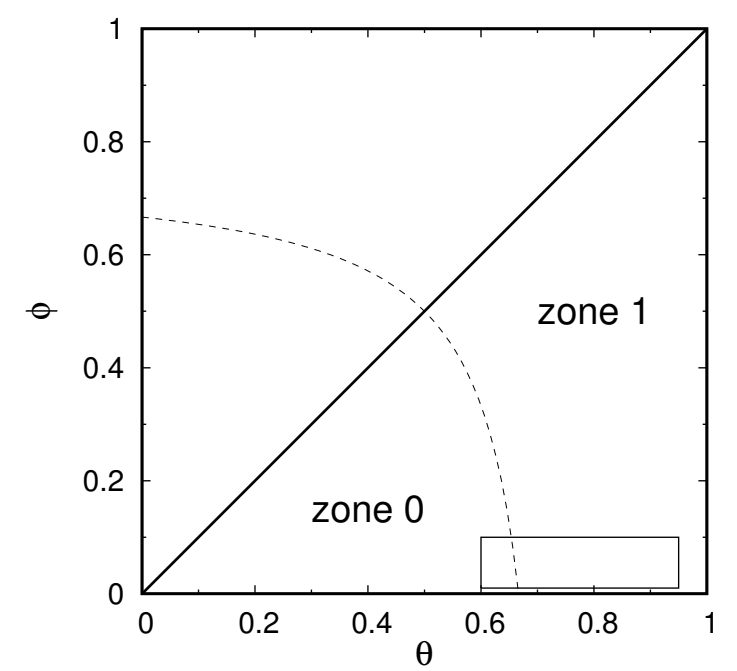

Figure 3.9: Zone 0 and I projected in the $\phi-\theta$ plane. The dashed line $\phi(\theta)=(2-3 \theta) /(3-4 \theta)$ separates the two zones. The enclosed region indicates the range of the parameter set $\mathrm{GH}$.

$0.001 \tilde{\omega}$. This may be because $\left|\omega_{x y 0}\right|=0.1 \tilde{\omega}$ is not small perturbation, and the spin might have escaped from the basin of attracter of Type SS behavior.

Lastly we consider the Type SW (spin wobbling) samples. The time when the spin starts to wobble roughly corresponds with $t_{r s}$ of Type $\mathrm{R}$ in Fig. 3.8; the center of wobbling $n_{w}$ and its amplitude vary from sample to sample. As in the case of Type $\mathrm{R}$, there are some samples which start to wobble after several times of $t_{r G H-}$ where $K_{r} \ll 0.1$. Wobbling behaviors of such samples are similar to those which start wobbling around $t_{r G H-}$. We remark that there are two qualitatively different Type SW behaviors. When $\left|n_{i}\right| \lesssim 0.4 \tilde{\omega}$, the spin of Type SW sample oscillates almost periodically. However, when $n_{i}=-0.5 \tilde{\omega}$ and $K_{r} \ll 0.1$, we find some samples that show "chaotic" oscillations as an example shown in Fig 3.7(b-3), which is similar to that observed in Fig 3.5(d). 


\section{Chapter 4}

\section{Summary and Discussion}

\subsection{Summary of our work}

We have performed the theoretical analysis and numerical simulations on the minimal model of rattleback. By reformulating Garcia and Hubbard's theory [16], we obtained the concise expressions for the asymmetric torque coefficients, Eqs. (2.44) and (2.45), gave the compact proof to the fact that the pitching and the rolling generate the torques with the opposite sign, and reduced the original dynamics to the three-variable dynamics by a physically transparent procedure.

Our expressions for the asymmetric torque coefficients are equivalent to those by Garcia and Hubbard, but we explicitly elucidate that the ratio of the two coefficient for the pitching and the rolling oscillation is proportional to the squared ratio of those frequencies. Since the pitching frequency is significantly higher than that of the rolling for a typical rattleback, the time for reversal to one spin direction (or unsteady direction) is much shorter than that to the other direction (or steady direction); the spin reversal for the latter direction is not usually observed in a real rattleback due to dissipation.

The simulations on the original dynamics for various parameter sets demonstrate that Garcia-Hubbard formulae for the first spin reversal time Eqs. (2.61) and (2.63) are good in the case of small initial spin and small oscillation for both the unsteady and the steady directions. The deviation from the formula is especially large for the steady direction in the fast initial spin and small $K_{r}$ regime, where the rattleback may not reverse and shows a variety of dynamics, that includes steady spinning, periodic and chaotic wobbling. 


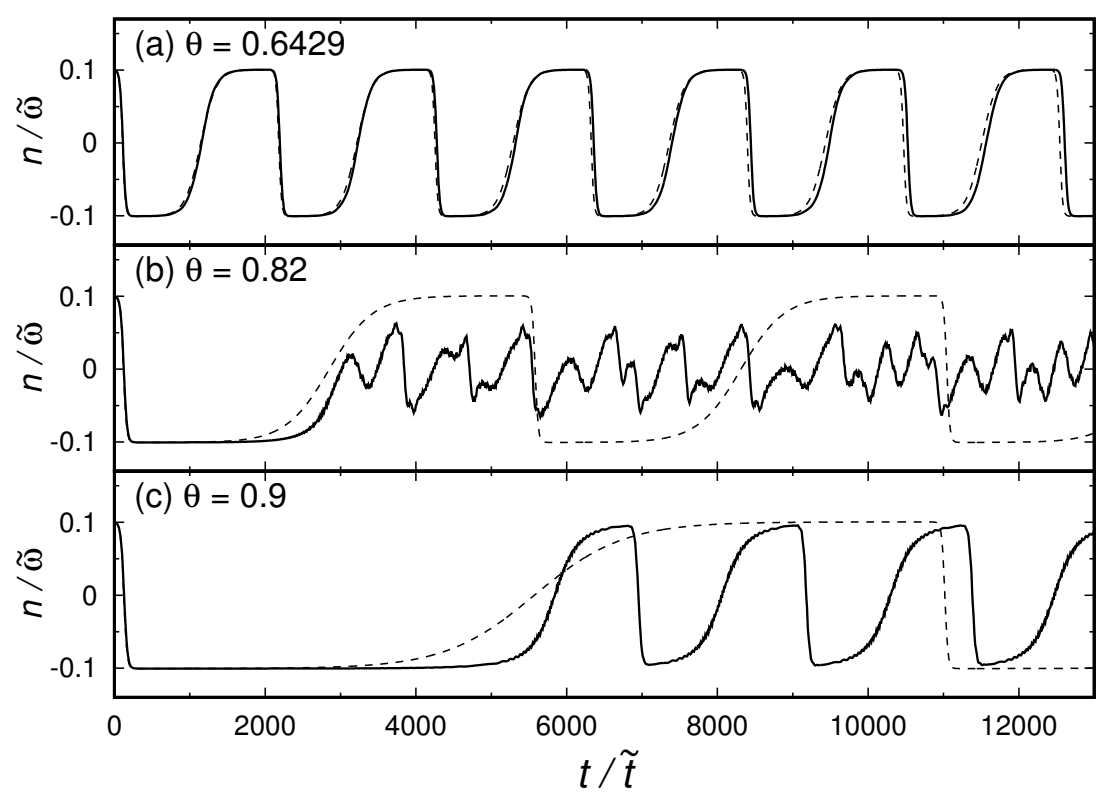

Figure 4.1: Three types of spin behaviors after the first reversal period in the small spin regime with $n_{i}=0.1 \tilde{\omega},\left|\omega_{x y 0}\right|=0.01 \tilde{\omega}$ for three different values of $\theta$, i.e. the curvature in the rolling direction. (a) A quasi-periodic behavior with $\theta=0.6429$ (the same with GH), (b) a chaotic behavior with $\theta=0.82$, (c) a quasi-periodic behavior with a period shorter than the first one with $\theta=0.9$. All the other parameters are the same as GH. The dashed lines show the spin evolutions for the corresponding simplified dynamics, where $\bar{E}_{p}(0)=M a^{2}\left[\alpha\left(\left|\omega_{x y 0}\right| \cos \psi_{p}\right)^{2}+\beta\left(\left|\omega_{x y 0}\right| \sin \psi_{p}\right)^{2}\right] / 2, \bar{E}_{r}(0)=3 \times 10^{-5} M a^{2} \tilde{\omega}^{2}$, and $\bar{n}(0)=n_{i}$.

\subsection{Discussions}

\subsubsection{After the first round of spin reversals}

In the present work, we study the minimal model for the rattleback dynamics, i.e., a spinning rigid body with a no-slip contact ignoring any form of dissipation. We have reduced the original dynamics to the simplified dynamics Eqs. (2.52)-(2.54) with the three variables. The assumptions and/or approximations used in the derivation are (i) the amplitudes of the oscillations are small, (ii) the coupling between the spin and the oscillations does not depend on the spin, and (iii) the time scale for the spin change is much longer than the oscillation periods. It is interesting to note that the last assumption is apparently analogous to that used in the derivation of an adiabatic invariant for some systems under slow change of an external parameter if the 
spin variable is regarded as a slow parameter. In the present case with this separation of time scales, the dynamics conserves the "Casimir invariant" $C$ of Eq. (2.56).

After the first round of spin reversals, our simplified dynamics Eqs. (2.52)(2.54) repeats itself and shows periodic behavior as well as the dynamics studied by Moffatt and Tokieda Eq. (2.94) because the system with only three variables has two conservative quantities, i.e., the total energy and the Casimir invariant. However, the Casimir invariant is an approximate one in the original dynamics, and invariant only under the approximations given at the beginning of this section. The Casimir "invariant" actually varies and the original system shows aperiodic behaviors.

A few examples for longer time evolutions of spin $n(t)$ are given in Fig. 4.1 for the system with the parameter set GH except for the curvature in the rolling direction $\theta=0.6429(\mathrm{GH}, \mathrm{a}), 0.82(\mathrm{~b})$ and 0.9 (c) along with those by the corresponding simplified dynamics. The first example (a) almost shows a periodic spin reversal behavior as is expected by the simplified dynamics. It is, however, only quasi-periodic with fluctuating periodicity. The second example (b) does not show a periodic behavior; the initial spin reversal till $t / \tilde{t} \approx 100$ is nearly the same with (a), but after the time of the second spin reversal around $t / \tilde{t} \approx 3000$, it turns into chaotic, deviating from the simplified dynamics. The third example (c) may look similar to (a) but is peculiar; it shows a quasi-periodic behavior after the initial round of spin reversals, and its periodicity is much shorter than that by the simplified dynamics.

The simplified dynamics seems to work reasonably well for the case of smaller $\theta$ in (a) but fails for larger $\theta$ close to 1 in (b) and (c). This indicates that the approximations or assumptions used to derive the simplified dynamics are not valid for the larger curvature in the rolling direction $\theta$; as the radius of curvature $1 / \theta$ becomes small and close to 1 , i.e., the height of the center of mass, the restoration force for the rolling oscillation becomes weak. This should result in the rolling oscillation with the larger amplitude and the slower frequency, thus the assumptions (i) and (iii) given at the beginning of this section may not be good enough.

The fact that the system shows a different behavior after the first round of spin reversals is reminiscent of the existence of attractors, which is normally prohibited in an energy-conserving system by Liouville theorem. In the present system, however, the theorem is invalidated by the non-holonomic constraint due to the no-slip condition Eq. (2.3). As mentioned already, the existence of strange attractors in an energy-conserving system with a nonholonomic constraint has been studied by Borizov et al. [24], and chaotic behavior in the rattleback system has been discussed in connection with the Casimir invariant by Yoshida et al. [43]. Yoshida et al. extended the 3-d 
dynamics by Moffatt and Tokieda Eq. (2.94) to a 4-d dynamics, where the Casimir "invariant" Eq. (2.100) slowly varies, and numerically observed the chaotic behavior. This may be related to the case of Fig. 4.1(b); for this case, the approximations that used in deriving the simplified dynamics are not valid, thus $C$ of Eq. (2.56) is not invariant anymore.

\subsubsection{Pippard's rattleback revisited}

Now let us revisit Pippard's rattleback in [6] we have introduced in Chapter 1, which shows more than one reversal before it stops. Assuming the long heavy bar determines the mass and the moments of inertia, the rattleback parameters are estimated as $\alpha=11.7, \beta=1.04, \gamma=10.8, \theta=0.67, \phi=$ 0.092 , and $\xi=6^{\circ}$. The corresponding asymmetric torque coefficients are $K_{p} \approx 1.42$ and $K_{r} \approx 0.56$; large $K_{p}$ and relatively large $K_{r}$ are consistent with his observation. Relatively large $K_{r}$ is attributed to $\theta=0.67$, which is significantly smaller than 1 , and small $\beta$; these two factors give fast rolling oscillation, which leads to relatively strong coupling between the rolling and the spinning.

\subsubsection{On elliptic and semi-elliptic rattlebacks}

We have shown in Section 2.3 that for typical rattlebacks time for reversal $t_{r G H-}$ diverges to infinity as $\theta \rightarrow 1$. For an ellipsoid or a semi-ellipsoid whose lower surface is given by $x^{2} / c^{2}+y^{2} / b^{2}+z^{2} / a^{2}=1, \theta$ is $a^{2} / c^{2}$ or $\left(5 a^{2}\right) /\left(8 c^{2}\right)$, respectively. Therefore, $t_{r G H-}$ is very sensitive to $a / c$ where the smaller radius of principal curvature $c^{2} / a$ is close to the height of the center of mass $a$ or $(5 a) / 8$. This shows that changing the height of the center of mass, for example by placing a weight somewhere at $z$-axis, can readily change the motion of the steady spinning direction.

We also remark that similar (semi-)elliptic rattlebacks have the same asymmetric coefficients. Garcia-Hubbard formulae Eqs. (2.61) and (2.63) indicate that the time for reversals are the same for such rattlebacks when initial spinning conditions $n_{i}$ and $n_{0}$ are the same. On the other hand, as a rattleback becomes larger, namely as the static height of the center of mass $a$ becomes larger, the pitch and roll periods become longer as can be seen from Eqs. (2.31) and (2.32). These unintuitive two results stem from the separation of the time scales between the time for reversal and the oscillation periods. As the rattleback size becomes larger, the assumption of the timescale separation becomes invalid at some point, thus the time for reversal changes. 


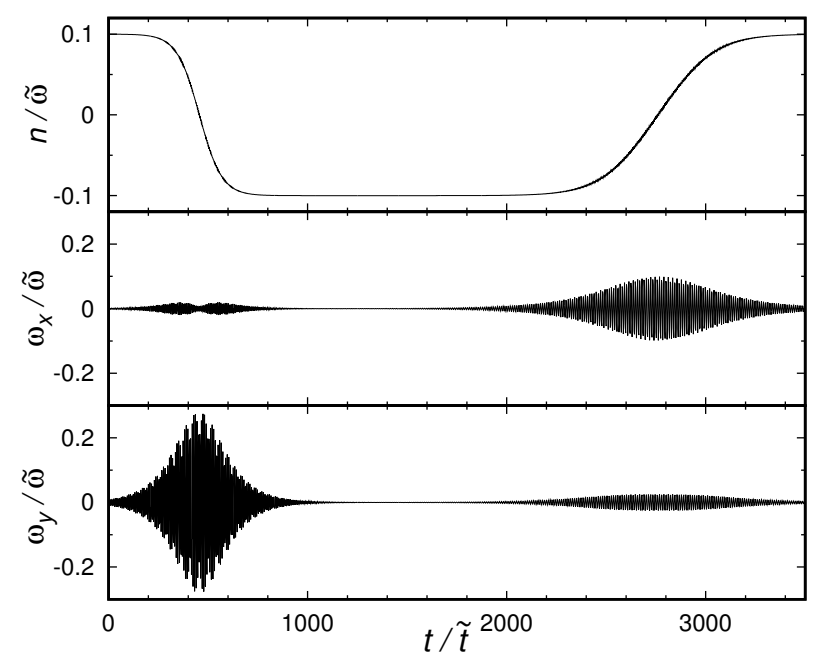

Figure 4.2: A spin evolution and the corresponding $\omega_{x}$ and $\omega_{y}$ for $\omega_{p 0}<\omega_{r 0}$. The parameter values are $\alpha=12, \beta=1.5, \gamma=12, \theta=0.5, \phi=0.2$, and $\xi=-3^{\circ}$, which gives $\omega_{p 0}^{2} / \omega_{r 0}^{2} \approx 1 / 2$.

\subsubsection{Atypical choices of parameters}

In this thesis, we have mainly considered the case where $|\xi| \ll 1$ with the pitch frequency being higher than the roll frequency, as in the case of usual rattlebacks. In this subsection, we briefly argue how the dynamics is modified if these restrictions are eased.

The expressions of the asymmetric torque coefficients Eqs. (2.44) and (2.45) are valid for "atypical" cases unless $\omega_{p} \approx \omega_{r}$. Therefore, the rattleback dynamics can be systematically studied by (i) specifying two oscillation modes, i.e., the eigenfrequencies and eigenvectors, and (ii) calculating the corresponding asymmetric torque coefficients. We remark that $K_{p} K_{r}>0$ holds for atypical choices of parameters, thus the averaged torques generated by the two oscillation modes always have opposite signs to each other.

As an example of atypical rattlebacks, let us consider the case of $\omega_{p 0}<\omega_{r 0}$ with $|\xi| \ll 1$, i.e., the pitching is slower than the rolling. In this case, $\omega_{p} \approx \omega_{r 0}$ and $\omega_{r} \approx \omega_{p 0}$ correspond to the rolling and the pitching modes, respectively, because $\omega_{p}>\omega_{r}$ by Eq. (2.28). Therefore, the spinning direction for which the pitching or the rolling is excited is changed, and the time for reversal due to the pitching becomes longer than that due to the rolling. A simulation result for the $\omega_{p 0}^{2} / \omega_{r 0}^{2} \approx 1 / 2$ case is shown in Fig. 4.2 , which should be compared with Fig. 3.4. As expected, the pitching develops when $n_{i}<0$ and the rolling develops when $n_{i}>0$, while the time for reversal is longer for the $n_{i}<0$ case than that for the $n_{i}>0$ case as in the case of $\omega_{p 0}>\omega_{r 0}$. 


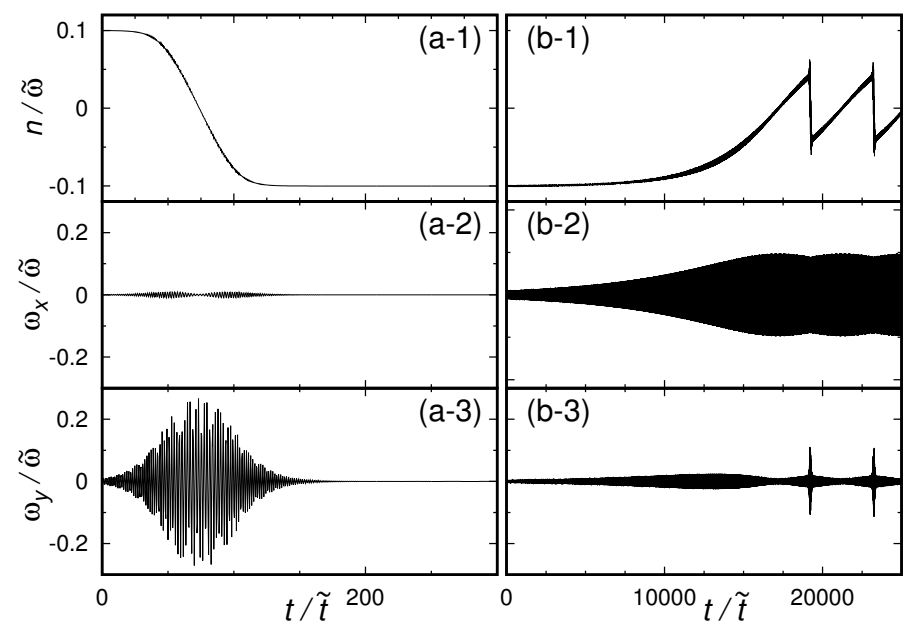

Figure 4.3: A spin evolution and the corresponding $\omega_{x}$ and $\omega_{y}$ for the skew angle $\xi=-85^{\circ}$, with initial spinning direction (a) $n_{i}>0$ and (b) $n_{i}<0$. All the other parameters are the same as GH. The directions of the eigenvectors are $\psi_{p} \approx-89.4^{\circ}$ and $\psi_{r} \approx 4.9^{\circ}$, and the corresponding asymmetric torque coefficients are $K_{p} \approx 1.3$ and $K_{r} \approx 0.003$.

As $|\xi|$ increases, the pitching and the rolling become ambiguous because the eigenvectors are not close to the geometrical axes anymore. As $|\xi|$ approaches $\pi / 2$, the two eigenvalues approach $\sqrt{(g / a)(1 / \phi-1) / \beta}$ and $\sqrt{(g / a)(1 / \theta-1) / \alpha}$, because exchanging $\phi$ and $\theta$, or $\alpha$ and $\beta$, corresponds to changing the skew angle by $\pi / 2$. Jeal Walker questioned what happens if the moment of inertia for the rolling becomes larger than that for the pitching, keeping the shape unchanged [2]; this corresponds to this "another small skewness" case, i.e., $|\xi| \approx \pi / 2$.

In Fig. 4.3 we show the simulation result for $\xi=-85^{\circ}$. The eigenvectors $\left|\omega_{p}\right\rangle$ and $\left|\omega_{r}\right\rangle$ are now close to the $y$-axis and $x$-axis, respectively, with

$$
\omega_{p}^{2} \approx\left(\frac{g}{a}\right) \frac{(1 / \phi-1)}{\beta} \text { and } \omega_{r}^{2} \approx\left(\frac{g}{a}\right) \frac{(1 / \theta-1)}{\alpha} .
$$

Thus the theory predicts that the pitching, mainly represented by $\omega_{y}$, develops when $n_{i}>0$, which is indeed observed in the simulation in Fig. 4.3. Note that $K_{r}$ is so small that the corresponding time for reversal is quite long, and it does not show a periodic behavior after the first reversal for $n_{i}<0$.

\subsubsection{On initial conditions}

When comparing the simulations with Garcia-Hubbard formulae, we have only considered the initial condition $\boldsymbol{u}(0)=(0,0,-1)^{t}$ with relevant oscil- 
lation. Treating other initial conditions, for example setting $\omega_{x 0}=\omega_{y 0}=0$ and giving $\boldsymbol{u}(0)$ and $\omega_{z 0}$, is remained for future work.

\subsection{Concluding remarks}

In conclusion, the rattleback dynamics is fascinating not only because it produces torques in an intriguing way, but also its non-holonomic nature shows surprisingly rich dynamics when dissipation is neglected. We believe that the rattleback system will keep on attracting physicist's attention. 


\section{Acknowledgment}

First of all, I am deeply grateful to my research adviser Prof. Hiizu Nakanishi for his invaluable support. I would like to thank all of our research group members, especially Prof. Kiyohide Nomura, Dr. Takahiro Sakaue, and Dr. Jun Matsui for helping my study. I also would like to thank Prof. Osamu Narikiyo and Prof. Yasuhide Fukumoto for the critical comments on my study.

Lastly I am grateful to my parents for their warm support, encouragement, and patience. 


\section{Appendix A}

\section{Rattleback parameters for semi-ellipsoids}

For an ellipsoid with a uniform mass density whose surface is given by

$$
\frac{x^{2}}{c^{2}}+\frac{y^{2}}{b^{2}}+\frac{z^{2}}{a^{2}}=1 \quad(b>c>a)
$$

it can be shown that the pitch frequency is always higher than the roll frequency as follows.

One can readily show that

$$
I_{x x}=\frac{M\left(b^{2}+a^{2}\right)}{5}, I_{y y}=\frac{M\left(c^{2}+a^{2}\right)}{5}, I_{z z}=\frac{M\left(c^{2}+b^{2}\right)}{5},
$$

thus, the inertial parameters $\alpha$ and $\beta$ of the ellipsoid are given by

$$
\alpha=\frac{b^{2}+6 a^{2}}{5 a^{2}}, \quad \beta=\frac{c^{2}+6 a^{2}}{5 a^{2}} .
$$

The lower surface around $(0,0, a)^{t}$ can be approximated as

$$
\frac{z}{a} \approx 1-\frac{x^{2}}{2 c^{2}}-\frac{y^{2}}{2 b^{2}}
$$

therefore $\theta$ and $\phi$ are given by

$$
\theta=a^{2} / c^{2}, \quad \phi=a^{2} / b^{2} .
$$

From Eqs. (A.3) and (A.5), we have

$$
\begin{aligned}
& \omega_{p 0}^{2}=\frac{g}{a} \frac{1 / \phi-1}{\alpha}=\frac{5 g}{a} \frac{(b / a)^{2}-1}{(b / a)^{2}+6}, \\
& \omega_{r 0}^{2}=\frac{g}{a} \frac{1 / \theta-1}{\beta}=\frac{5 g}{a} \frac{(c / a)^{2}-1}{(c / a)^{2}+6} .
\end{aligned}
$$


Since $b / a>c / a>1$ and $f(x)=\left(x^{2}-1\right) /\left(x^{2}+6\right)$ is a monotonously increasing function where $x>1, \omega_{p 0}^{2}>\omega_{r 0}^{2}$ is shown.

For a semi-ellipsoid whose lower surface is described by Eq. (A.1) with the origin being the same as the ellipsoid, the height of the center of mass is (5/8)a.

The radii of principal curvature are the same as the ellipsoid, thus we have

$$
\theta=\frac{5 a}{8} \frac{a}{c^{2}}=\frac{5 a^{2}}{8 c^{2}}, \quad \phi=\frac{5 a^{2}}{8 b^{2}},
$$

where $b^{2}>c^{2}>5 a^{2} / 8$. The expressions of the moments of inertia about the axes of symmetry $I_{x x}^{\prime}, I_{y y}^{\prime}$, and $I_{z z}^{\prime}$ are the same as the ellipsoid if we use $M$ as the total mass. By using the parallel axis theorem, we obtain

$$
I_{x x}=I_{x x}^{\prime}-M\left(\frac{3 a}{8}\right)^{2}=\frac{M b^{2}}{5}+\frac{19}{320} M a^{2}, \quad I_{y y}=\frac{M c^{2}}{5}+\frac{19}{320} M a^{2} .
$$

Then $\alpha, \beta$ are given by

$$
\alpha=\frac{b^{2}}{5(5 a / 8)^{2}}+\frac{19}{320(5 / 8)^{2}}+1=\frac{b^{2}}{A a^{2}}+B, \quad \beta=\frac{c^{2}}{A a^{2}}+B,
$$

where $A=5^{3} / 8^{2}, B=\left(19+5^{3}\right) / 5^{3}$. Note that $A B=9 / 4$.

From Eqs. (A.8) and (A.10), $\omega_{p 0}$ and $\omega_{y 0}$ are given by,

$$
\begin{aligned}
& \omega_{p 0}^{2}=\frac{g}{a} \frac{1 / \phi-1}{\alpha}=\frac{g}{a} \frac{\frac{8 b^{2}}{5 a^{2}}-1}{\frac{1}{A}\left(\frac{b^{2}}{a^{2}}+A B\right)}=\frac{g}{a} \frac{25}{8} \frac{\frac{b^{2}}{a^{2}}-\frac{5}{8}}{\frac{b^{2}}{a^{2}}+\frac{9}{4}} \\
& \omega_{r 0}^{2}=\frac{g}{a} \frac{1 / \theta-1}{\beta}=\frac{g}{a} \frac{25}{8} \frac{\frac{c^{2}}{a^{2}}-\frac{5}{8}}{\frac{c^{2}}{a^{2}}+\frac{9}{4}} .
\end{aligned}
$$

Since $f(x) \equiv\left(x^{2}-5 / 8\right) /\left(x^{2}+9 / 4\right)$ is a monotonously increasing function for $x^{2}>5 / 8, \omega_{p 0}^{2}>\omega_{r 0}^{2}$ is shown.

As stated in Section 2.4, Zone II does not exist if $I_{z z}-I_{x x}>0$. In the case of semi-ellipsoids,

$$
I_{z z}-I_{x x}=M a^{2}\left(\frac{1}{5} \frac{c^{2}}{a^{2}}-\frac{19}{320}\right)>0,
$$

thus Zone II also does not exist for semi-ellipsoidal rattlebacks. 


\section{Appendix B}

\section{Equivalence of $K_{p}$ and $K_{r}$ with the original expressions}

Garcia and Hubbard [16] calculated the asymmetric torque coefficients using the eigenvalue equation in $\left|\omega_{\perp}\right\rangle$-space, i.e.,

$$
\begin{aligned}
\left|\ddot{\omega}_{\perp}\right\rangle & =-\frac{g}{a} \hat{J}^{-1}(\hat{\Gamma}-1)\left|\omega_{\perp}\right\rangle \\
& =-\frac{g}{a}\left(\begin{array}{cc}
\left(\Gamma_{11}-1\right) / \alpha, & \Gamma_{12} / \alpha \\
\Gamma_{12} / \beta, & \left(\Gamma_{22}-1\right) / \beta
\end{array}\right)\left|\omega_{\perp}\right\rangle \\
& \equiv-\left(\begin{array}{cc}
\Lambda_{11}, & \Lambda_{12} \\
\Lambda_{21}, & \Lambda_{22}
\end{array}\right)\left|\omega_{\perp}\right\rangle .
\end{aligned}
$$

The eigenvalues $\omega_{p}$ and $\omega_{r}$ are the same as those of Eq. (2.26), and are given by

$$
\omega_{p, r}^{2}=\frac{1}{2}\left[\left(\Lambda_{11}+\Lambda_{22}\right) \pm \sqrt{\left(\Lambda_{11}-\Lambda_{22}\right)^{2}+4 \Lambda_{12} \Lambda_{21}}\right] .
$$

The original expressions of asymmetric torque coefficients by Garcia and Hubbard can be written in our notation as

$$
\begin{aligned}
& K_{p G H}=\frac{\Gamma_{12}(1 / \beta-1 / \alpha) \omega_{p}^{2}\left(\omega_{p}^{2}-\Lambda_{22}\right)}{\left(\omega_{p}^{2}-\Lambda_{22}\right)^{2}+\Lambda_{12} \Lambda_{21}}, \\
& K_{r G H}=\frac{\Gamma_{12}(1 / \beta-1 / \alpha) \omega_{r}^{2}\left(\Lambda_{11}-\omega_{r}^{2}\right)}{\left(\Lambda_{11}-\omega_{r}^{2}\right)^{2}+\Lambda_{12} \Lambda_{21}} .
\end{aligned}
$$

From Eq. (B.2), following relations hold:

$$
\begin{aligned}
\omega_{p}^{2}-\Lambda_{22} & =\Lambda_{11}-\omega_{r}^{2}=\frac{1}{2}\left[\left(\omega_{p}^{2}-\omega_{r}^{2}\right)+\left(\Lambda_{11}-\Lambda_{22}\right)\right] \\
\Lambda_{12} \Lambda_{21} & =\frac{1}{4}\left[\left(\omega_{p}^{2}-\omega_{r}^{2}\right)^{2}-\left(\Lambda_{11}-\Lambda_{22}\right)^{2}\right]
\end{aligned}
$$


which gives

$$
\left(\omega_{p}^{2}-\Lambda_{22}\right)^{2}+\Lambda_{12} \Lambda_{21}=\frac{1}{2}\left(\omega_{p}^{2}-\omega_{r}^{2}\right)\left[\left(\omega_{p}^{2}-\omega_{r}^{2}\right)+\left(\Lambda_{11}-\Lambda_{22}\right)\right] .
$$

Eqs. (B.3)-(B.7) immediately give our expressions of the asymmetric torque coefficients $K_{p}$ and $K_{r}$ by Eqs. (2.44) and (2.45). 


\section{Bibliography}

[1] G. T. Walker. On a dynamical top. Q. J. Pure Appl. Math., Vol. 28, pp. $175-184,1896$.

[2] J. Walker. The mysterious "rattleback": a stone that spins in one direction and then reverses. Sci. Am., Vol. 241, pp. 172-184, 1979.

[3] H. Crabtree. An Elementary Treatment of the Theory of Spinning Tops and Gyroscopic Motion. Longmans, Green and Co., 1909.

[4] V. D. Berger and M. G. Olsson. Classical Mechanics A Modern Perspective. McGraw-Hill, Inc., 1973.

[5] 時枝正. それでも帰ってくる軌道. 数学セミナー 2015 年 1 月号, pp. 2-5, 2015. in Japanese.

[6] A. B. Pippard. How to make a celt or rattleback. Eur. J. Phys., Vol. 11, pp. 63-64, 1990.

[7] G. T. Walker. On a curious dynamical property of celts. Proc. Cambr. Phil. Soc., Vol. 8, pp. 305-306, 1895.

[8] A pen portrait of Sir Gilbert Walker. http://www .walker-institute. ac.uk/about/sir_gilbert.htm.

[9] H. Bondi. The rigid body dynamics of unidirectional spin. Proc. R. Soc. Lond. A, Vol. 405, pp. 265-274, 1986.

[10] M. Wakasugi. カイラリテイを持つ岡体の方向選択的回転. Master's thesis, Tokyo Univ., 2011. In Japanese.

[11] W. Case and S. Jalal. The rattleback revisited. Am. J. Phys., Vol. 82, pp. 654-658, 2014.

[12] A. P. Markeev. On the dynamics of a solid on an absolutely rough plane. Prikl. Mat. Mekh., Vol. 47, pp. 575-582, 1983. 
[13] M. Pascal. Asymptotic solution of the equations of motion for a celtic stone. Prikl. Mat. Mekh., Vol. 47, pp. 321-329, 1983.

[14] A. D. Blackowiak, R. H. Rand, and H. Kaplan. The dynamics of the celt with second order averaging and computer algebra. In Proceedings of the ASME Design Engineering Technical Conferences, Sacramento, California, 1997.

[15] H. K. Moffatt and T. Tokieda. Celt reversals: a prototype of chiral dynamics. P. Roy. Soc. Edinb. A, Vol. 138, pp. 361-368, 2008.

[16] A. Garcia and M. Hubbard. Spin reversal of the rattleback: theory and experiment. Proc. R. Soc. Lond. A, Vol. 418, pp. 165-197, 1988.

[17] T. R. Kane and D. A. Levinson. Realistic mathematical modeling of the rattleback. Int. J. Nonlin. Mech., Vol. 17, pp. 175-186, 1982.

[18] R. E. Lindberg, Jr. and R. W. Longman. On the dynamic behavior of the wobblestone. Acta Mech., Vol. 49, pp. 81-94, 1983.

[19] A. Nanda, P. Singla, and M. A. Karami. Energy harvesting using rattleback: Theoretical analysis and simulations of spin resonance. J. Sound. Vib., Vol. 369, pp. 195-208, 2016.

[20] A. V. Borisov and I. S. Mamaev. Strange attractors in rattleback dynamics. Phys. Usp., Vol. 46, pp. 393-403, 2003.

[21] A. V. Borisov, A. A. Kilin, and I. S. Mamaev. New effects in dynamics of rattlebacks. Dokl. Phys., Vol. 51, pp. 272-275, 2006.

[22] H. R. Dullin and A. V. Tsygvintsev. On the analytic non-integrability of the rattleback problem. Ann. Fac. Sci. Toulouse Math., Vol. 17, pp. 495-517, 2008.

[23] A.V. Borisov, A. Y. Jalnine, S. P. Kuznetsov, I. R. Sataev, and J. V. Sedova. Dynamical phenomena occurring due to phase volume compression in nonholonomic model of the rattleback. Regul. and Chaotic Dyn., Vol. 17, pp. 512-532, 2012.

[24] A. V. Borisov, A. O. Kazakov, and S. P. Kuznetsov. Nonlinear dynamics of the rattleback: a nonholonomic model. Phys. Usp., Vol. 57, pp. 453460, 2014. 
[25] A. S. Gonchenko, S. V. Gonchenko, and A. O. Kazakov. Richness of chaotic dynamics in nonholonomic models of a celtic stone. Regul. Chaotic Dyn., Vol. 18, pp. 521-538, 2013.

[26] K. Magnus. Zur Theorie der Keltischen Wackelsteine. Z. Angew. Math. Mech., Vol. 54, pp. 54-55, 1974.

[27] A. V. Karapetyan. On realizing nonholonomic constraints by viscous friction forces and celtic stones stability. Prikl. Mat. Mekh., Vol. 45, pp. 42-51, 1981.

[28] H. Takano. Spin reversal of a rattleback with viscous friction. Regul. Chaotic Dyn., Vol. 19, pp. 81-99, 2014.

[29] V. Ph. Zhuravlev and D. M. Klimov. Global motion of the celt. Mech. Solids, Vol. 43, pp. 320-327, 2008.

[30] J. Awrejcewicz and G. Kudra. Celtic stone dynamics revisited using dry friction and rolling resistance. Shock Vib., Vol. 19, pp. 1115-1123, 2012.

[31] G. Kudra and J. Awrejcewicz. Approximate modelling of resulting dry friction forces and rolling resistance for elliptic contact shape. Eur. J. Mech. A-Solid, Vol. 42, pp. 358-375, 2013.

[32] G. Kudra and J. Awrejcewicz. Application and experimental validation of new computational models of friction forces and rolling resistance. Acta Mech., Vol. 226, pp. 2831-2848, 2015.

[33] H. K. Moffatt. Euler's disk and its finite-time singularity. Nature, Vol. 404, pp. 833-834, 2000.

[34] M. A. Jalali, M. S. Sarebangholi, and M. Alam. Terminal retrograde turn of rolling rings. Phys. Rev. E, Vol. 92, 032913, 2015.

[35] H. K. Moffatt and Y. Shimomura. Spinning eggs - a paradox resolved. Nature, Vol. 416, pp. 385-386, 2002.

[36] H. K. Moffatt, Y. Shimomura, and M. Branicki. Dynamics of an axisymmetric body spinning on a horizontal surface. I. stability and the gyroscopic approximation. Proc. R. Soc. Lond. A, Vol. 460, pp. 3643$3672,2004$.

[37] Y. Shimomura, M. Branicki, and H. K. Moffatt. Dynamics of an axisymmetric body spinning on a horizontal surface. II. self-induced jumping. Proc. R. Soc. Lond. A, Vol. 461, pp. 1753-1774, 2005. 
[38] T. Mitsui, K. Aihara, C. Terayama, H. Kobayashi, and Y. Shimomura. Can a spinning egg really jump? Proc. R. Soc. Lond. A, Vol. 462, pp. 2897-2905, 2006.

[39] M. Heckel, P. Müller, T. Pöschel, and J. A. C. Gallas. Circular ratchets as transducers of vertical vibrations into rotations. Phys. Rev. E, Vol. 86, 061310, 2012.

[40] Y. Kubo, S. Inagaki, M. Ichikawa, and K. Yoshikawa. Mode bifurcation of a bouncing dumbbell with chirality. Phys. Rev. E, Vol. 91, 052905, 2015 .

[41] M. Bächer, E. Whiting, B. Bickel, and O. Sorkine-Hornung. Spin-it: optimizing moment of inertia for spinnable objects. In ACM Transactions on Graphics (TOG) - Proceedings of ACM SIGGRAPH 2014, Vol. 33, 2014.

[42] H. Goldstein, C. Poole, and J. Safko. Classical Mechanics (3rd edition). Addison Wesley, 2002.

[43] Z. Yoshida, T. Tokieda, and P. J. Morrison. A prototype rattleback model - a Lie-Poisson Bianchi Type VI system with chirality. arXiv:1609.09223v1 [math-ph], 2016. 